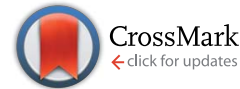

Cite this: RSC Adv., 2014, 4, 63296

\title{
Graphene and its nanocomposite material based electrochemical sensor platform for dopamine
}

\author{
Alagarsamy Pandikumar, $\dagger^{\star a}$ Gregory Thien Soon How, $\dagger^{a}$ Teo Peik See, ${ }^{a}$ \\ Fatin Saiha Omar, ${ }^{a}$ Subramaniam Jayabal, ${ }^{a}$ Khosro Zangeneh Kamali, ${ }^{a}$

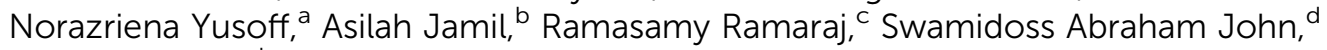 \\ Hong Ngee Lim ${ }^{\text {be }}$ and Nay Ming Huang*a
}

Dopamine (DA) is an important catecholamine neurotransmitter in the mammalian central nervous system that influences several physiological functions. The impact of DA levels within the human body significantly affects the body functions. Maintaining DA level is essential and the electrochemical detection methods are often used to detect the DA level to regulate the body function. In this review, graphene (functionalized graphene and $\mathrm{N}$-doped graphene) and its composites (metal, metal oxide, polymer, carbonaceous materials, clay, zeolite, and metal-organic framework based graphene composites) modified electrodes with their improved sensing performance towards DA along with several interfering species are described. Further, recent developments on the fabrication of various graphene based composite modified electrodes are also presented. Some important strategies to improve the selectivity and sensitivity towards DA with graphene based composite modified electrodes are also described.

Received 4th November 2014 Accepted 10th November 2014

DOI: $10.1039 / c 4 r a 13777 a$

www.rsc.org/advances

\section{Introduction}

${ }^{a}$ Low Dimensional Materials Research Centre, Department of Physics, Faculty of Science, University of Malaya, 50603 Kuala Lumpur, Malaysia. E-mail: pandikumarinbox@gmail.com; huangnayming@um.edu.my

${ }^{b}$ Department of Chemistry, Faculty of Science, Universiti Putra Malaysia, 43400 Serdang, Malaysia

'School of Chemistry, Centre for Photoelectrochemistry, Madurai Kamaraj University, Madurai-625021, India

${ }^{d}$ Centre for Nanoscience \& Nanotechnology, Department of Chemistry, Gandhigram Rural University, Gandhigram-624302, India

${ }^{\circ}$ Functional Device Laboratory, Institute of Advanced Technology, Universiti Putra Malaysia, 43400, Serdang, Malaysia

$\dagger$ These two authors contributed equally to this work.
Neurotransmitters are endogenous chemicals that transmit signals across a synapse from one neuron cell to another 'target' cell. ${ }^{1}$ Acting as the chemical messengers of the nervous system, neurotransmitters relay information across synapses via excitation or inhibition of the next neuron or effector tissue. ${ }^{2}$ Neurotransmitters are mainly classified into amino acids, peptides, and monoamines. The amino acids are glutamate, aspartate, D-serine, $\gamma$-aminobutyric acid, and glycine. The monoamines are histamine, adrenaline, dopamine (DA),

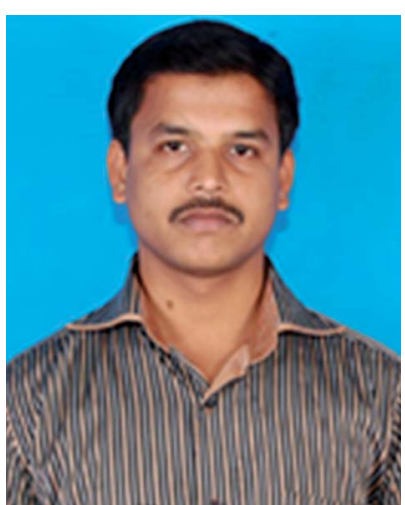

Alagarsamy Pandikumar received his B.Sc. and M.Sc. degrees from Gandhigram Rural University in the year 2004 and 2006, respectively. He received his Ph.D. degree (2014) in Chemistry from Madurai Kamaraj University. Currently, he is working as a High Impact Research (HIR) - Post Doctoral Research Fellow in University of Malaya. His current research interests include graphenebased metal and metal oxide nanocomposites for photocatalysis, photoelectrochemical cells, dye-sensitized solar cells, and sensor applications.

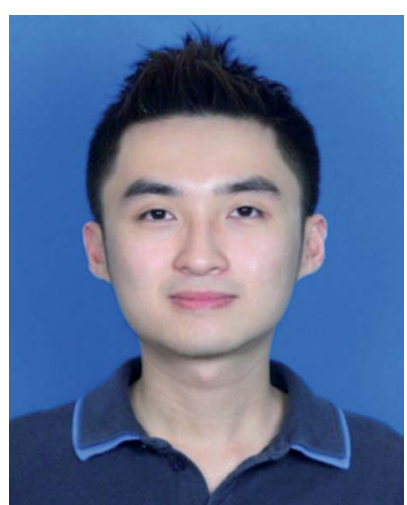

Gregory Thien Soon How received his B.Sc. in Physics from University of Malaya in the year 2011. He is currently a graduate M.Sc. student under the supervision of Dr Nay Ming Huang in the Low Dimensional Material Research Centre (LDMRC), Department of Physics, University of Malaya. His current research interest lies in graphene based nanomaterials for environmental and sensing applications such as photocatalysis, solar cells, and chemical sensors. 
noradrenaline, serotonin, and melatonin. Peptides include somatostatin, cocaine, and opioid. ${ }^{3}$ The other neurotransmitters also include acetylcholine, adenosine, anandamide, nitric oxide, etc. Monoamine neurotransmitters are neurotransmitters and neuromodulators that contains one amino group connected with an aromatic ring by a two-carbon chain $\left(-\mathrm{CH}_{2}-\mathrm{CH}_{2}-\right)$. All the monoamine neurotransmitters are synthesized from aromatic amino acids like phenylalanine, tyrosine, tryptophan, and thyroid hormones by the action of aromatic amino acid decarboxylase enzymes. Monoamines are produced in the axon terminal of the neuron and transported to the axon terminal, where they are packaged into synaptic vesicles and stored. ${ }^{4}$ The monoamine neurotransmitters play an important role in the secretion and production of neurotrophin3 by astrocytes, an important local cellular source of trophic support in the normal and also diseased brain. ${ }^{5}$

\section{Dopamine: an important neurotransmitter}

Neurotransmitters are the endogenous substance that allows transmission of nerve impulses between neurons through synapse. Often classified as excitatory or inhibitory based on the behaviour

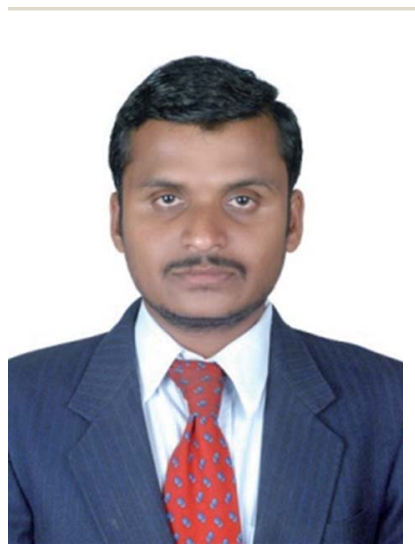

Subramaniam Jayabal received his M.Sc. (2007) and M.Phil. (2008) degrees in Chemistry from Madurai Kamaraj University. In 2014, he has submitted his Ph.D. thesis to Madurai Kamaraj University under the supervision of Prof. R. Ramaraj for the award of his PhD degree in Chemistry. Currently, he is working with Dr Huang Nay Ming as a research assistant in University of Malaya. His research interest focuses on the synthesis of nanostructured materials for sensor applications.

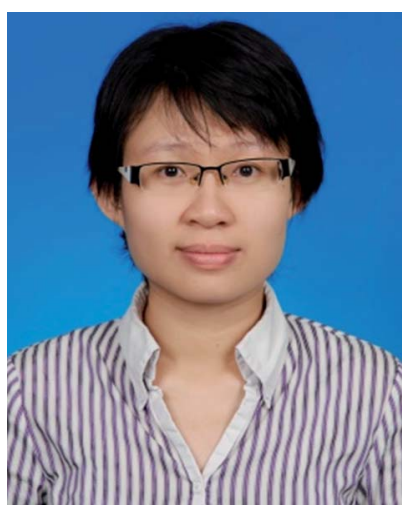

Hong Ngee Lim received her B.Sc. and M.Sc. degrees from Universiti Kebangsaan Malaysia in year 2002 and 2004, respectively. She was awarded a Ph.D. degree in Chemistry from Universiti Putra Malaysia in 2010. Her research experience involves working as an Assistant Professor at the Nottingham University Malaysia Campus and as a Senior Lecturer in the University of Malaya before joining Universiti Putra Malaysia as a Senior Lecturer. Since 2009, she has been actively involved in graphene-related research, mainly in encompassing the synthesis of graphene-based nanomaterials and their applications. of neurons, two have the opposite effect whereby inhibitory neurotransmitters calm the brain and excitatory neurotransmitters stimulate the brain. In the neurotransmission process, DA acts as an extrasynaptic messenger molecule via volume transmission across the synaptic cleft to bind to extrasynaptic receptors ( $G$ protein-coupled receptors) and transporters. ${ }^{5,6}$ However, DA is a unique neurotransmitter as it possesses both excitatory and inhibitory classification. It's vital function lies in regulating attention, cognition, pleasure, movement, and hormonal processes. ${ }^{7}$ In addition, it is widely distributed in the central nervous, renal, hormonal, and cardiovascular systems. ${ }^{8}$

\section{Impact of dopamine level in human body}

The abnormality in the amount of DA produced in the central nervous system is a sign of an underlying condition of a neurological disorder in the human body. Low level of DA has been strongly related with illness such as Parkinson's disease, restless leg syndrome, attention deficit hyperactivity disorder (ADHD), schizophrenia, and HIV infection. ${ }^{9-12}$ Moreover, DA is also highly linked with the reward mechanism in the brain. Since DA acts as a neurotransmitter which is vital for message transfer functions, the use of illegal drugs or substance abuse such as heroin, cocaine, nicotine, and alcohol blocks the DA transport that inhibits the re-uptake of DA and eventually increases the DA levels, causing an increased risk of depression and drug addiction. ${ }^{13}$ Therefore, finding a reliable analytical method is important in order to evaluate the disease progress.

\section{Conventional detection methods for dopamine}

In the past few decades, various analytical methods have been established and reported for the detection of DA (Fig. 1). This includes the capillary electrophoresis mass spectrometry

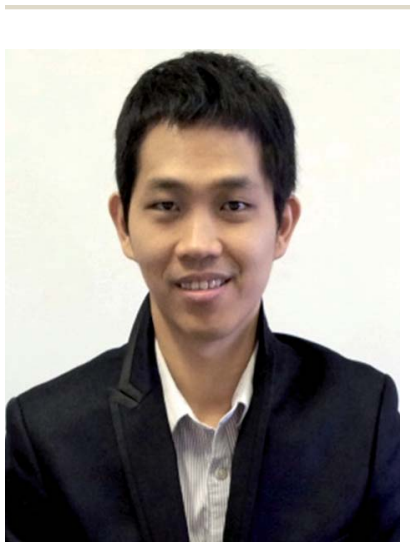

Nay Ming Huang received his B.Sc., M.Sc., and Ph.D. degrees from Universiti Kebangsaan Malaysia. He joined University of Malaya in 2009 as a Senior Lecturer in the Department of Physics, Faculty of Science. His current research interests involve graphene and graphene based nanocomposites for sensor, solar energy conversion, and energy storage applications. He is the recipient of the National Young Scientist Award (2012) by the Ministry of Science, Technology and Innovation Malaysia, MASS Young Scientist Award (2012) by the Malaysia Association of Solid States, and also the UM Young Researcher Award (2012) by University of Malaya. Currently, he has published more than 50 papers on graphene related research work. 


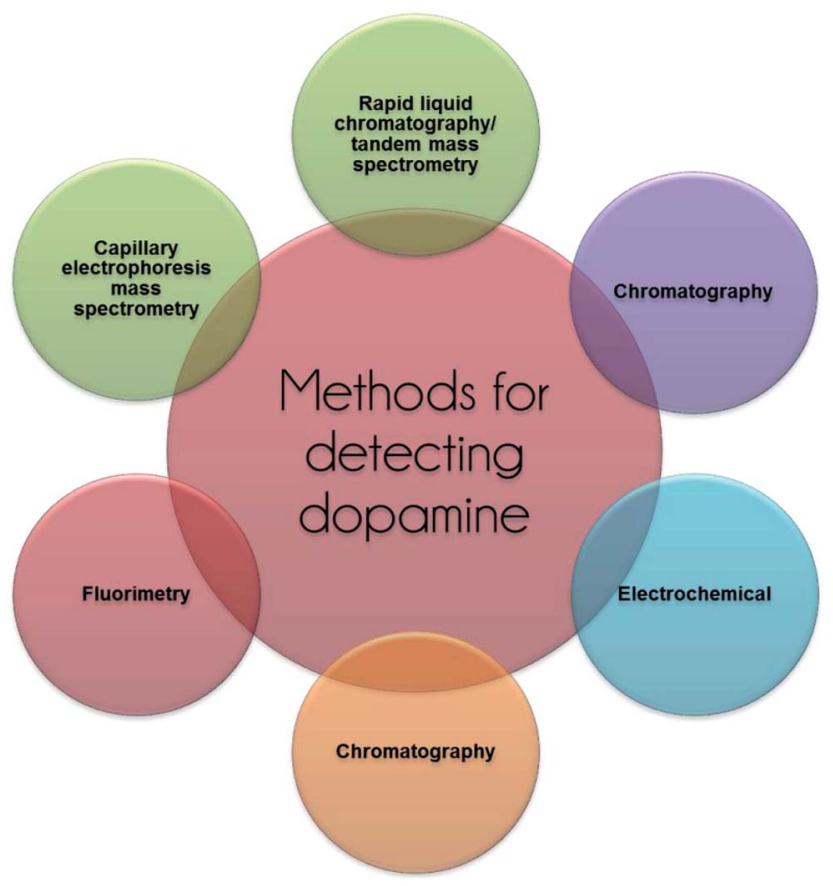

Fig. 1 Various analytical methods for the detection of dopamine.

method, chromatography, fluorimetry, rapid liquid chromatography/tandem mass spectrometry (LC-MS/MS), chemiluminescence. ${ }^{14-16}$ Although these methods provide high sensitivity in the detection of DA, they have several drawbacks. They require a complex system, long time-consumption, highcost, and take a substantial large amount of workspace. Hence, the detection of DA by electrochemical method is a more suitable approach in evaluating the low concentration of DA under physiological conditions. This method provides beneficial characteristics of simplicity, short time delay, cost-effective, good selectivity, and real-time detection without comprising its sensitivity capabilities. ${ }^{17-19}$

\section{Principle of an electrochemical sensor}

Generally, chemical modification poses a greater use than biological substrates in the detection of DA. This is due to the fact that biological substrates incur a fouling effect which can be attributed to the reaction products accumulating and forming electro-polymerized films. ${ }^{20}$ Electrochemical sensors find its use in wide applications such as glucose monitors and ion sensors which are mainly applied in the solid-state chemical sensors field. ${ }^{21}$ An electrochemical sensor is able to produce an electrical output signal into digital signal for further analysis through a series of principal stages as shown in Fig. 2. Basically, the response obtained in electrochemical sensors is due to the interaction between chemistry and electricity which are based on potentiometric, amperometric, and conductivity measurements. ${ }^{22}$ In the fabrication of chemically modified electrochemical sensors, the detection of DA takes into consideration

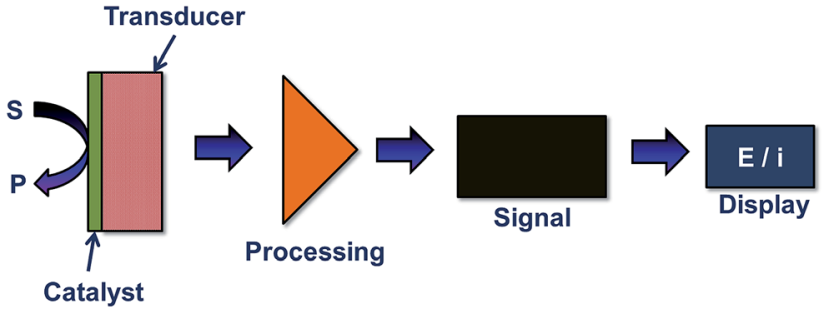

Fig. 2 Principal stages in the operation of an electrochemical sensor.

of two main factors; the enhancement in electrocatalytic activity and the selectivity of DA with several potential interfering species.

\section{Challenges in electrochemical dopamine sensor}

It is well known that DA is an electroactive compound which can be electrochemically oxidized to form dopamine-quinone (DAQ) at the electrode surface. When a potential is applied on the electrode, an interchange of 2 electrons and 2 protons occurs in the DA compound and is then oxidized to DAQ. Hence, a faradaic current signal is produced when the electrode received the electrons. ${ }^{23}$ Traditionally, conventional electrochemical method was widely used in the direct determination of DA. However, the poor response signal was often observed when using conventional electrodes, caused by the sluggish electron-transfer rate properties at the electrode's surface. ${ }^{24}$ Besides, the fouling effect occurring at the bare electrode limits the sensitivity and selectivity capabilities towards DA detection. ${ }^{25}$ Further, the co-existence of various interfering species such as ascorbic acid (AA) and uric acid (UA) causes the overlapping voltammetric response that results in difficulties in distinguishing the individual peak potentials. ${ }^{26}$ Hence, an effective way to resolve this issue is by surface modification technique of the working electrode to circumvent the overvoltage and slow kinetics of the electrode process (Fig. 3 ). ${ }^{27}$ Through this, achieving a suitable modification to obtain high sensitivity and selectivity towards DA sensors is the ultimate goal.
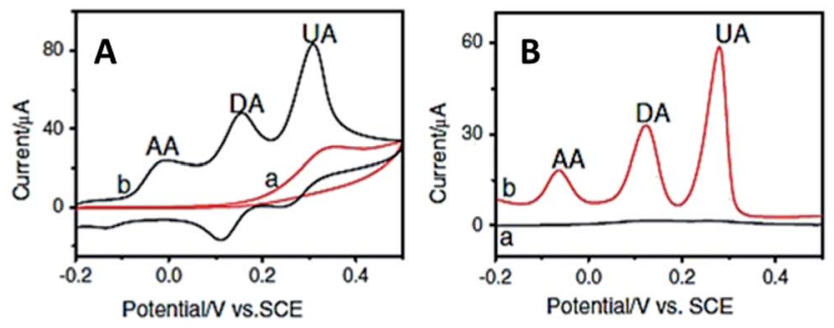

Fig. 3 (A) Cyclic voltammograms and (B) DPV curves obtained for (a) bare $G C$ and chemically modified electrode in the presence of DA, AA and UA. 


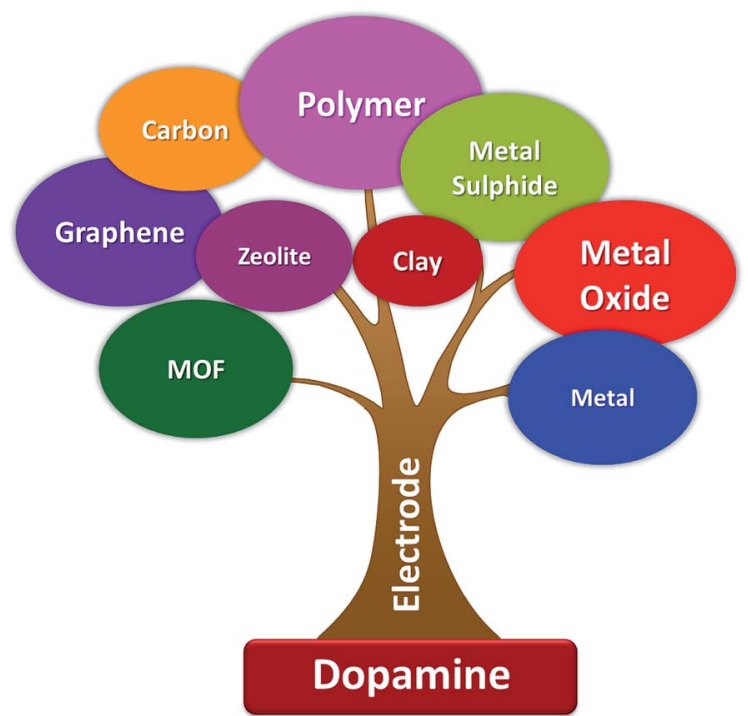

Fig. 4 Various electrode materials used for the detection of dopamine.

\section{Overview of modified electrodes for dopamine}

The chemically modified electrodes are often used to detect DA with high selectivity and sensitivity. The most commonly used electrode active materials are metal nanoparticles ( $\mathrm{Au}, \mathrm{Ag}, \mathrm{Pt}, \mathrm{Pd}$, $\mathrm{Cu}){ }^{28-31}$ metal oxides $\left(\mathrm{TiO}_{2}, \mathrm{Fe}_{2} \mathrm{O}_{3}, \mathrm{Fe}_{3} \mathrm{O}_{4}, \mathrm{SnO}_{2}, \mathrm{CuO}, \mathrm{ZnO}\right),{ }^{32-37}$ polymers (Nafion, chitosan, $o$-phenylenediamine, polyaniline, polypyrrole), ${ }^{38-42}$ carbon nanotubes, ${ }^{43-45}$ zeolite $^{46}$ clay, ${ }^{47}$ and metal-organic frameworks (MOF) ${ }^{48}$ (Fig. 4). In terms of the analytical techniques used in the electrochemical detection of DA, various electrochemical techniques were used which include cyclic voltammetry (CV), ${ }^{49}$ linear sweep voltammetry (LSV), ${ }^{50,51}$ differential pulse voltammetry (DPV), ${ }^{52}$ square wave voltammetry $(\mathrm{SWV}){ }^{53}$ chronoamperometry (CA), ${ }^{54}$ electrochemiluminescence (ECL),$^{55}$ impedimetric, ${ }^{56}$ and transistor-gate. ${ }^{57}$

\section{Graphene-an ultimate electroactive material for biosensors}

Graphene is a 2D sheet of $\mathrm{sp}^{2}$ bonded carbon atoms, densely packed in a honeycomb crystal lattice structure whereby each of these layers is held together by weak van der Waals forces. ${ }^{58}$ The most important property of graphene is its excellent electrical conductivity. The various forms of graphene-based materials include thermally reduced graphene oxide (TRGO), chemically reduced graphene oxide (CRGO), and electrochemically reduced graphene oxide (ERGO), contains oxygen-containing functional groups and certain amounts of defects. ${ }^{59-61}$ The rapid electron transfer takes place at the surface of edge planes and defects when compared to the basal planes for the electrochemical sensors fabricated with graphene based materials. ${ }^{62-64}$ The presence of these structural defects in the chemically modified graphene can be exploited for electrochemical sensor applications.

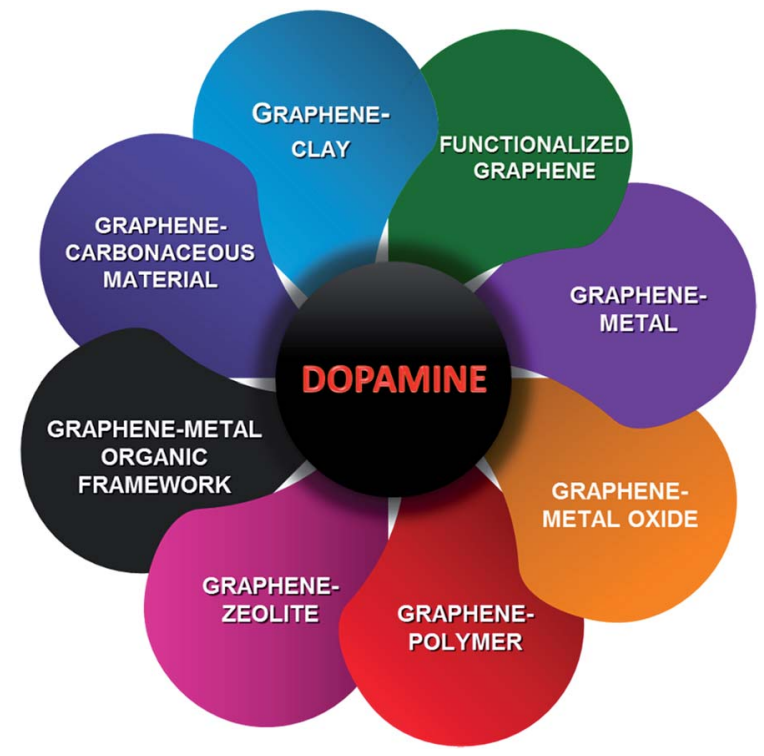

Fig. 5 Various graphene and their composites used for the electrochemical detection of dopamine.

The presence of oxygen-containing functional groups in the graphene-based materials play a vital role in the electrochemical sensors, which makes the adsorption and preconcentration of the redox species (which is of our analytical interest) and effectively catalyse the redox reactions. In addition, the presence of these functional groups makes an effective functionalization with various biomolecules and polymers for applications. ${ }^{65-67}$ The functionalization of these graphene based materials with specific functional groups can enable the use of these excellent materials for electrochemical sensor applications with specific analytes. The functionalized graphene materials also make fast electron transfer by pre-concentrating the target analytes at the electrode surface. Besides DA, graphene-based materials are also used for electrochemical sensing of other biomolecules such as UA, AA, glucose, cholesterol, folic acid, tryptophan, serotonin and L-dopa. ${ }^{68-72}$ Among these biomolecules, DA is the most important biomolecule (more details given in Sections 2 and 3) and it is more often used to be detected by graphene (functionalized and $\mathrm{N}$ doped) and their composites with metals, metal oxides, metal-organic frameworks, polymers, clay, zeolite, and carbonaceous materials (Fig. 5).

\section{Graphene and their composite based electrochemical dopamine sensor}

Making composite with metals, metal oxides, metal organic frameworks, polymers, clay, zeolite, and carbonaceous materials, graphene acquires excellent electrocatalytic properties which lead to the better detection of DA in the presence of interfering species. At first, graphene was used as the electrode modifier for the detection of DA in 2009 due to its unique electrochemical and physiochemical properties. ${ }^{73}$ This report 
initiates the new development for the detection of DA by graphene-based composite materials with metal, ${ }^{74}$ metal oxides, ${ }^{75}$ and polymer $^{76}$ in the year 2010 with improved sensitivity and electrocatalytic activity. In 2011, graphene-carbonaceous materials and graphene-clay composites were used as alternative electrode modifier materials for the detection of DA. ${ }^{77,78}$ Other than composite materials, the role of functionalized graphene for the detection of DA was also investigated in the same year. ${ }^{79}$ Following this, a new field emerges in understanding $\mathrm{N}$-doped graphene materials for the detection of $\mathrm{DA}^{\mathbf{8 0}}$ in 2012. Recently (2014), MOF with porous and large surface area was incorporated into graphene materials that open up a new possibility for the development of sensitive DA sensors (Fig. 6). ${ }^{81}$

\subsection{Graphene as electrochemical sensor}

The 2D hexagonal lattice graphene emerged as an ideal candidate who has captured enormous interests and grown explosively in electrocatalysis and electrochemical biosensors. The $\mathrm{sp}^{2}$ hybridization, unique electronic configuration, its extraordinary electron transport property, so called ballistic conductivity, and the number and position of the oxygen functionalities of graphene helps to promote and accelerate the electron transfer process during electrochemical detection. However, the ability of rapid electron transfer kinetics at the basal planes of graphene sheets act as nanoconnectors and thus electrically facilitates the heterogeneous electron transfer between the biomolecules and electrode substrate, resulting in excellent sensing performance. In comparison to CNT, Pumera et al. proved that graphene displays better conductivity, stability, sensitivity, and significantly exhibiting faster heterogeneous electron transfer rate, which is attributed to the nanographite impurities. ${ }^{82}$ Gao and co-workers ${ }^{83}$ reported the fabrication of an eco-friendly electrochemical sensor based on graphene oxide modified electrode (GO/GCE) by covalent coupling method for simultaneous determination of DA and AA (Fig. 7). The different electrochemical parameters and the impact on the peak response of DA and AA were evaluated at this modified electrode. In contrast to the other DA biosensors based on chemically reduced graphene materials, this work not only demonstrated better stability and reproducibility, but also

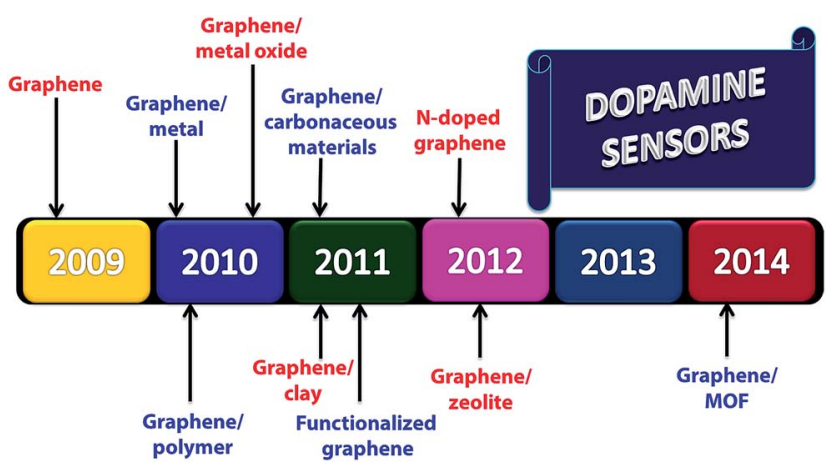

Fig. 6 Timeline for the development of graphene and their nanocomposite materials based electrochemical detection of dopamine.

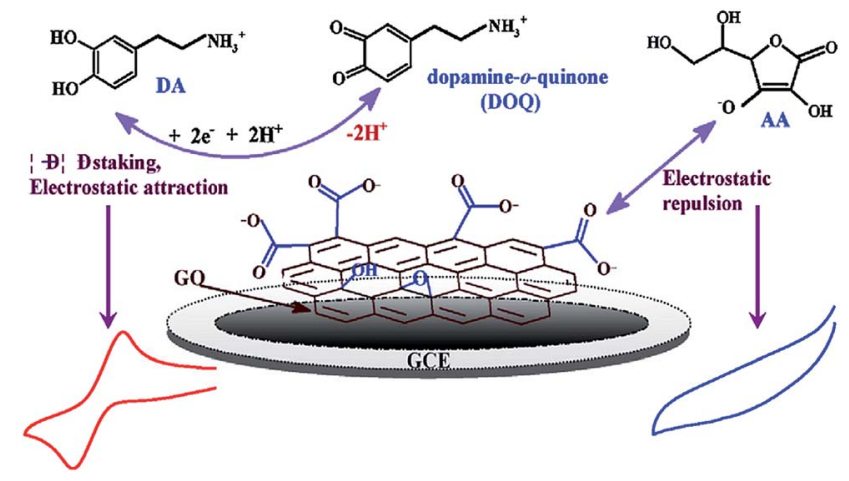

Fig. 7 Different electrochemical pathway for DA and AA on the GO/ GCE surface. Reprinted with permission from ref. 73, Copyrights (2013) Elsevier Publications.

showed highly selective redox response towards DA in the concentration ranging from 1 to $15 \mu \mathrm{M}$ with a limit of detection (LOD) of $0.27 \mu \mathrm{M}$. The special phenyl structure with delocalized $\pi$ electrons in GO efficiently interacts with the aromatic ring of DA that are strengthen through $\pi-\pi$ stacking forces. On the other hand, the electrostatic repulsion between GO and AA causes the complete suppression of AA oxidation (Fig. 7).

Similarly, Wang et $a l^{73}$ and Kim et $a l .{ }^{84}$ fabricated an electrochemical sensor using the graphene modified electrode for the sensitive and selective detection of DA. The graphenemodified electrodes displayed better electrochemical performance than MWCNTs modified electrode for selective determination of DA in large excess of AA and was completely eliminating the interference effect of AA. This remarkable increased performance of graphene to DA and AA may be correlated to the elusive $2 \mathrm{D}$ structure and unusual electronic properties of graphene. Additionally, the $\pi-\pi$ interactions between the aromatic structure of graphene and DA molecules accelerates faster electron transfer rate and at the same time, weakens the AA oxidation at the modified electrode.

Wang and co-workers ${ }^{85}$ fabricated a facile DA biosensor based on macroporous flower-like graphene-nanosheets cluster (f-RGO) modified glassy carbon electrode (GCE) surface. The obtained modified electrode portraying a large surface area, may provide more active sites for DA oxidation owing to the increased electrolyte-accessible surface area of f-RGO, fast electron transfer process, and the enhancement of DA electrocatalytic performance with good discrimination between DA, $\mathrm{AA}$, and UA in comparison with the bare GCE and layer-stacking RGO/GCE. Yang et al. $^{26}$ exploited the electrochemically reduced graphene oxide film on GCE (ERGO/GCE) for the simultaneous determination of three electro-active species, namely DA, AA, and UA. The fabricated modified electrode contains unique structural features including high electroactive surface sites and numerous edge plane defects of ERGO favourable for the oxidations of DA, AA, and UA. Hence, this electrode showed reliable recovery for DA in urine samples, indicating that the ERGO is a promising candidate in sensing and biosensing applications. Additionally, Yang et al. ${ }^{\mathbf{8 6}}$ proposed an ERGO on carbon fiber electrode (CFE) to investigate the electrocatalytic 
activity towards AA, DA, and UA. The obtained low detection limit for DA $(0.77 \mu \mathrm{M})$ suggests that the ERGO/CFE showed high sensitivity and selectivity towards simultaneous determination of these biomolecules. Similarly, Zhang et $a .^{87}$ demonstrates that the partially electrochemical reduced graphene oxide (pERGO) modified electrode showed a significantly high electrocatalytic activity and LOD for the detection of DA which could be attributed to its relatively low background current. In a different study, Raj and $\mathrm{John}^{\mathbf{8 8}}$ reported the fabrication of electrochemically reduced graphene oxide by electrochemical reduction of electrostatically assembled GO on 1,6-hexadiamine (HDA) linker (Fig. 8(A)). The authors claimed that the present modification of ERGO shows advantages as the process does not involve any electrochemical pre-treatment of GCE. Moreover, the amine groups of HDA on the GCE surface does not easily desorbed at a more negative potential. Thus, the ERGO modified electrode displays low surface fouling effect, high stability, and increased electrochemical response towards DA oxidation (Fig. 8(B) and (C)).

Recently, $\mathrm{Yu}$ et $a .^{89}$ reported a three-dimensional (3D) interpenetrating graphene electrode (ErGO) fabricated by the electrochemical reduction of GO for selective detection of DA. The merit of this investigation is that the ErGO electrode was used directly without further functionalization or blending with other functional materials. This electrode can efficiently bring down the oxidation potential of AA to lower potential, thus facilitates to selectively detect DA in the presence of AA and UA. The ErGO modified sensor electrode exhibited a linear response in the concentration range of $0.1-10 \mu \mathrm{M}$ with a LOD of $0.1 \mu \mathrm{M}$ DA. Furthermore, this ErGO electrode exhibits good reproducibility and environmental stability, and was used for real sample analysis. Liu et al. ${ }^{90}$ reported a DA sensor based on graphene-nanosheets paste (GNSP) with the existence of cetyltrimethylammonium bromide (CTAB) for the selective detection $\mathrm{DA}$ and AA. In this work, the CTAB acts as a discriminating agent to minimize the fouling effect at the electrode by protecting micelles on the surface of graphene nanosheets. In comparison with graphite paste electrode, GNSP showed enhanced currents for DA and AA which may be related to the promotion of electron transfer by large density of edge/defects sites in the graphene nanosheets and the formation of micelle on the surface of GNS from CTAB aggregation. Hence, the discriminating effect of surfactant in the amplification feature of GNSP greatly improved the sensitivity and selectivity towards DA detection in the presence of interference species.

Nancy et $a l .{ }^{91}$ developed a new strategy for the synthesis of graphene by solar exfoliation method. The resulted solar exfoliated graphene modified electrode (sG/GCE) is utilized for the voltammetric resolution and determination of DA, AA, and UA. Interestingly, the use of binder-free sG/GCE effectively resolved the three electroactive species into well-defined peaks with high potential separations, which was mainly corresponded to the existence of enriched edge planes and oxygenated defects which was demonstrated in the Raman and FTIR analysis in SG sheets. Ying and co-workers ${ }^{92}$ introduced a novel, disposable, and sensitive screen-printed electrode (SPE) based on ionic liquid doped screen-printing ink which contains graphene (SPGNE) to investigate the electrochemical response of DA, AA, and UA. The screen-printing ink which contains graphite and cellulose acetate, dominates the electrocatalytic performance of SPEs

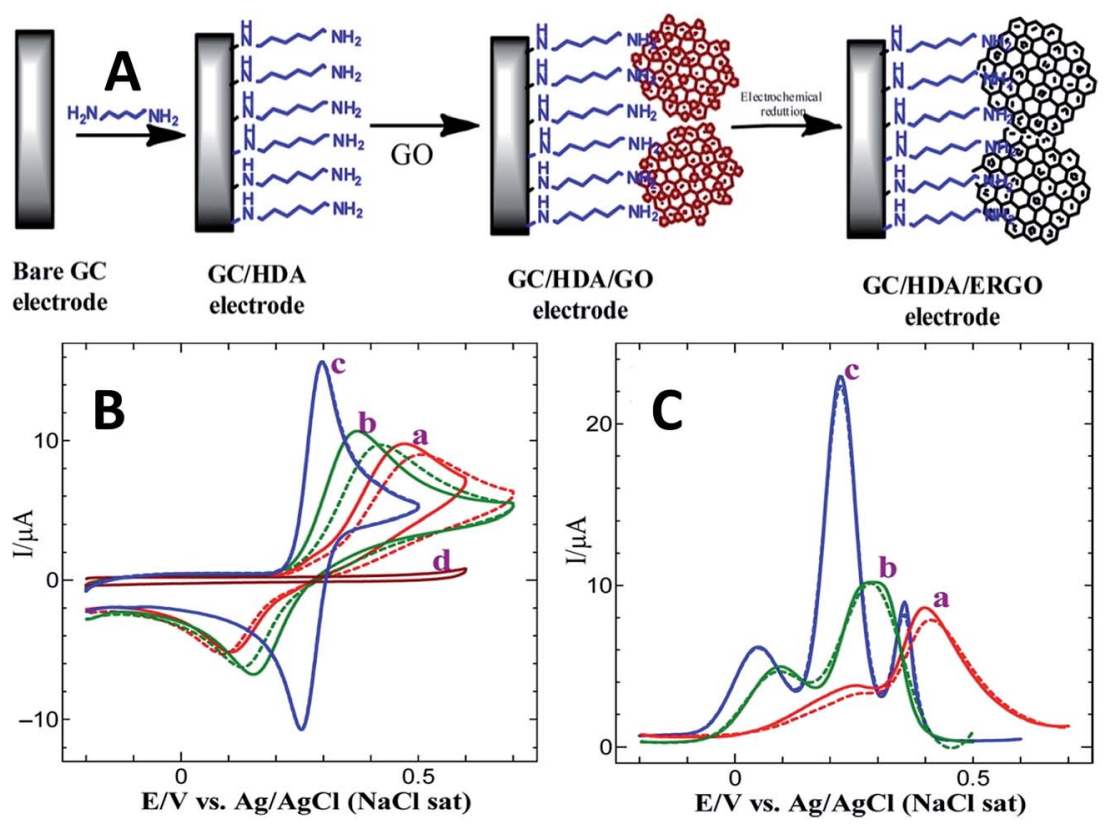

Fig. 8 (A) Schematic illustration for the fabrication of ERGO modified GCE, (B) CVs obtained for the oxidation of $0.5 \mathrm{mM}$ of DA at (a) bare GC, (b) $\mathrm{GC} / \mathrm{HDA} / \mathrm{GO}$, and (c) GC/HDA/ERGO electrodes in $0.2 \mathrm{M} \mathrm{PB}$ solution (pH 5.5) at a scan rate of $50 \mathrm{mV} \mathrm{s}^{-1}$. Solid line: first cycle; dotted line: eighth cycle. (C) DPVs obtained for the oxidations of each $0.5 \mathrm{mM}$ of AA, DA, and UA at (a) bare GCE, (b) GC/HDA/GO, and (c) GC/HDA/ERGO electrodes in 0.2 M PB solution. Solid line: first cycle; dotted line: eighth cycle. Reprinted with permission from ref. 79, Copyrights (2013) American Chemical Society. 
towards different analytes. The developed graphene-based ink electrode coupled with the advantageous properties of graphene and SPE that displayed rich of edge-plane defective sites, shows good antifouling ability, high stability and reproducibility, and rapid electron transfer kinetics which consequently results in the excellent electrochemical performance and well-resolved determination of the co-existing electroactive compounds (DA, $\mathrm{AA}$, and UA).

Recently, Huang et al. ${ }^{93}$ reported a electrochemical DA sensor with 3D graphene foam (GF). In this study, a three-dimensional macroporous and highly conductive GF was synthesized at atmospheric pressure using chemical vapour deposition (CVD). And then, the sensor electrode was fabricated by transferring the CVD produced GF onto the ITO glass and used for electrochemical detection of DA and AA. The GF electrode exhibits high selectivity for the detection of DA and AA. It also shows high sensitivity for detection of DA with the LOD $\sim 2.0 \mathrm{nM}$ in the linear response up to $\sim 10 \mathrm{mM}$. Furthermore, the 3D GF electrode was used for real samples, drug injection solutions, and human blood serum. Similarly, Yu et al. ${ }^{94}$ introduced a 3D reduced graphene oxide (3D-rGO) for DA sensor using polystyrene (PSs) as sacrificial templates through a templateassisted self-assembly method. The advantages of the high surface area and the open pore structure of 3D-rGO hinders the aggregation of rGO in aqueous solution, thus leading to a better sensing performance in the selective determination of DA and it showed LOD of $0.17 \mu \mathrm{M}$ with the linear range of $5 \mu \mathrm{M}-1 \mathrm{mM}$. The 3D-rGO/GCE showed good stability (97.7\% of its initial current response to DA after 10 days storage) and also it shows good reproducibility with RSD of $5.5 \%$ and repeatability with the RSD of $4.6 \%$. Hence, it is proven to be an effective functional material candidate in a wide range of applications. Meanwhile, Shang et al. ${ }^{95}$ demonstrated an efficient and rapid growth of uniform graphene nanoflake films (MGNFs) with sharp edges on Si substrates by substrate-lift-up method using microwaveassisted plasma enhanced chemical vapour deposition (MPCVD) for simultaneous determination of DA, AA, and UA. They established clearly that the novel edge-plane-based electrochemical interface reveals fast electron-transfer (ET) kinetics, active and sensitive electrocatalytic activity which is superior to all bare solid-state (carbon based) electrode, and is only comparable to the edge plane pyrolytic graphite (EPPG) electrode. The significantly enhanced sensing properties of MGNFs are mainly contributed to the special nest-like morphology, large surface area, and edge plane sites/defects acting as the nanoconnectors that can transport the electrons to the underneath substrate.

For the first time, Zhang et al. ${ }^{\mathbf{9 6}}$ developed a novel and sensitive sensing device for DA based on solution-gated graphene transistors (SGGTs) with functionalized gate electrodes. In this work, the semimetal with zero band-gap of graphene function as both channel and gate electrodes, portraying a stable performance and high sensitivity with a detection limit down to $1 \mathrm{nM}$ in DA determination which is three times much better than that of conventional electrochemical measurements. The authors indicated that the novel sensing mechanism of SGGTs is mainly due to the electrochemical oxidation of
DA at the graphene gate electrode which leads to the change of effective gate voltage applied on the transistor (Fig. 9). Besides that, the surface modification on the graphene gate electrode can further improved the sensitivity and selectivity of the sensing device in electroanalysis of different biomolecules.

The demonstration of the practical application for the graphene modified electrodes with the quantitative determination in the real samples (human blood serum, urine) by standard addition method is a crucial step in order to study the reliability and applicability of the proposed detection procedure. The recovery results obtained from the spiked samples was found in the range between $97-105 \%$ with a small relative standard deviation (R.S.D) in the electrochemical methods. This reflects the effect-free of the interference species (AA, UA, proteins) in the determination of DA and the potential application of graphene modified electrodes for analysing the real samples.

The reported graphene based DA sensors exhibited high sensitivity and excellent selectivity towards the determination of DA. The electrochemical sensing devices introduced by Zhang et al. ${ }^{96}$ Huang et al. ${ }^{93}$ and Raj and John, ${ }^{88}$ presents relatively low LODs of DA concentration. Especially in the whole graphene solution-gated graphene transistors (SGGTs) reported by Zhang et al. ${ }^{\mathbf{9 6}}$ for the first time it was shown a different working principle from the typical electrochemical instruments. The sensing performance is three times better than conventional electrochemical methods with the detection limit achieving down to $1 \mathrm{nM}$. The high sensitivity of SGGTs is mainly contributed to the interaction between the analytes and graphene channel, leading to the change of effective gate voltage applied on the transistor in the electro-oxidation of DA at the graphene gate electrodes. Thus, the excellent sensitivity, easy to dispose, and flexible whole-graphene SGGT device can be applied as a potential biosensor in the sensing platform.

\section{2. $\mathbf{N}$-doped-graphene as electrochemical sensor}

The intrinsic chemical doping with heteroatoms on graphene is an effective way for modulating and tailoring the surface chemistry, structural, and physico-chemical properties of graphene. This is beneficial in which it opens up new possibilities or provide fundamental insight into doped-graphene materials for electrochemistry applications such as providing efficient pathways for fast electron transfer and the introduction of a band gap. ${ }^{97}$ Nitrogen is considered as a potential candidate and excellent element for chemical doping attributed to its comparable atomic size and five valence electrons that are able to form strong valence bonds with carbon atoms. Substitution with electron-rich atom, such as nitrogen may lead to an increase in free charge carriers in the graphene's aromatic ring network and therefore leading to an enhanced conductivity and tuning of band gap. ${ }^{\mathbf{9 8}, 99}$ Hence, the $\mathrm{N}$-doped graphene is believed to be used for graphene modification and has a great potential in the applications of electrocatalysis and biosensors. ${ }^{\mathbf{1 0 0}}$

Li and co-workers ${ }^{\mathbf{1 0 1}}$ prepared an electrochemical DA sensor using $\mathrm{N}$-doped graphene (NG) synthesized by rapid thermal expansion-exfoliation and covalent transformation from melamine (MA)-graphene oxide (GO) mixture (Fig. 10). They studied 
(a)

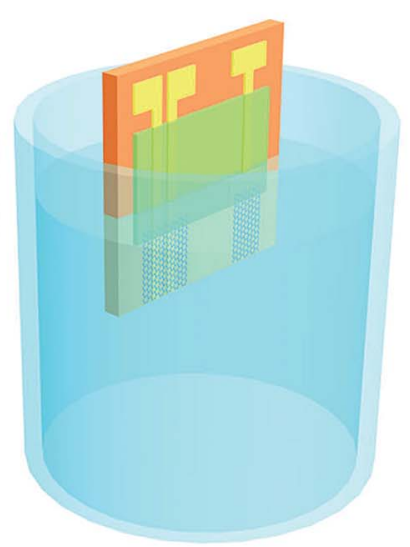

(b)

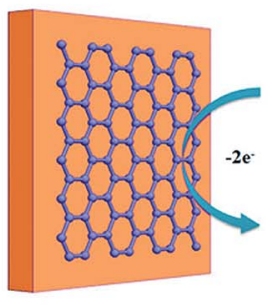

Gate electrode

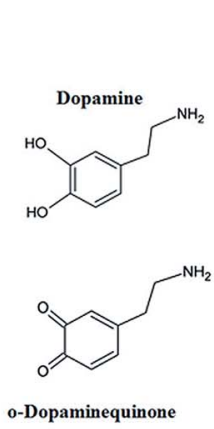

o-Dopaminequinone

(c)
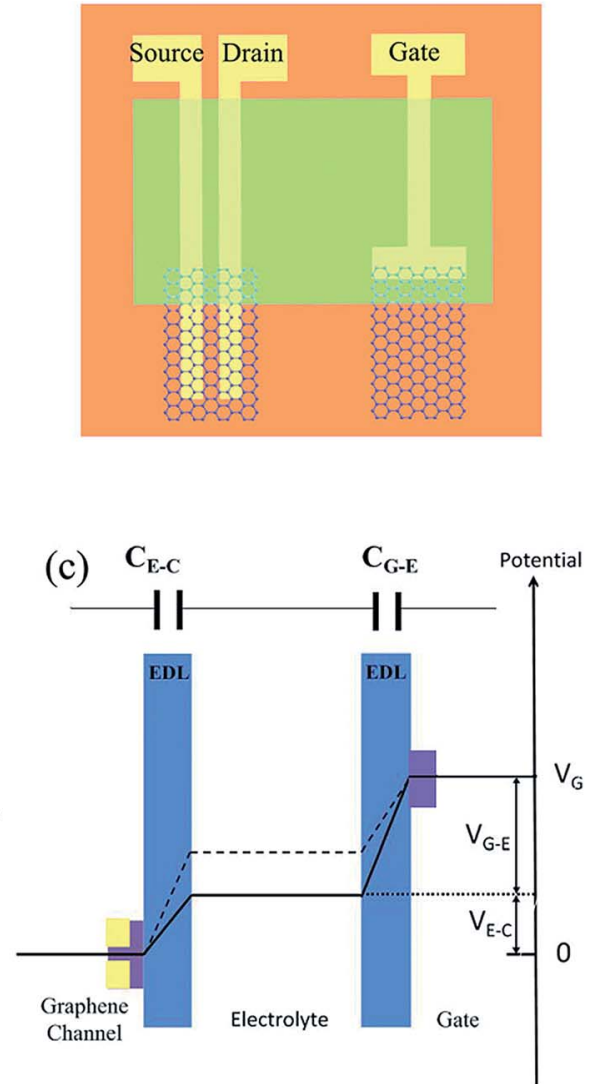

Glass/PET

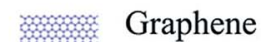

$\mathrm{Au}$

Silicone

Fig. 9 (a) Schematic diagram of a SGGT with graphene channel and a graphene gate electrode characterized in electrolyte. (b) Electrochemical reaction of dopamine on the graphene gate electrode. (c) Potential distribution between the gate and the channel of a SGGT in electrolyte. Reprinted with permission from ref. 86, Copyrights (2014) John Wiley and Sons.

the relationship of the $\mathrm{N}$-doped configuration (i.e. pyridinic-N, pyrrolic-N and graphitic-N), specific surface area, and electrocatalytic activity towards DA, AA, and UA on the fabricated NGs. Surprisingly, NG modified electrode showed significantly enhanced catalytic activity and excellent electrochemical sensitivity towards simultaneous determination of DA, AA, and UA which is mainly attributed to the large specific area, mesoporous structures, and N doping sites possessed by NG. A larger specific area of NG may provide more N-doping sites for DA oxidation. Furthermore, the formation of strong hydrogen bonds between the pyrrolic-N structure in NG and DA molecules strengthen the interaction between DA and $\mathrm{N}$-doped functional groups and negatively shifts the oxidation peak potentials of DA, AA, and UA. Similarly, Sheng and co-workers ${ }^{80}$ also introduced $\mathrm{N}$-doped graphene fabricated via thermal annealing of GO and melamine mixture. The synthesized NG used for determining AA, DA, and UA shows enhanced electrocatalytic responses towards oxidation of these species. The increased electrocatalytic activity could be attributed to the unique structure and properties of NG and interaction of hydrogen bond and $\pi-\pi$ stack between NG and target biomolecules. Additionally, Li et al. ${ }^{102}$ successfully developed a modified electrode using $\mathrm{N}$-doped graphene and polyethylenimine (PEI) based on electrostatic layer-by-layer (LBL) self-assembly method. The study demonstrates that the assembled NG multilayer films exhibits fast electron transfer and enhanced current response signals towards oxidation of DA.

Among the reported N-doped graphene based DA sensors, the $\mathrm{N}$-doped graphene prepared via thermal annealing of graphene with melamine precursor reported by Sheng and co-workers ${ }^{80}$ displayed the lowest LOD towards DA sensing. ${ }^{80,101,102}$ The excellent electrocatalytic performance of NG is mainly attributed to its unique 2D geometric microstructure and properties. The presence of $\pi-\pi$ interaction between the aromatic ring of DA molecules and the formation of hydrogen bonds between nitrogen atoms within graphene layers and hydroxyl or amine group in DA molecules, contribute to the enhanced electron transfer kinetics and subsequently decrease the overpotential of biomolecules (AA, DA, UA) oxidation according to their interaction modes. The doping level of pyridine nitrogen in NG samples could tailor the electronic properties in NG and further influenced the electrocatalytic activity of graphene in DA oxidation. Therefore, the NG nanosheets appear as an ideal candidate for a sensitive and selective biosensor. 


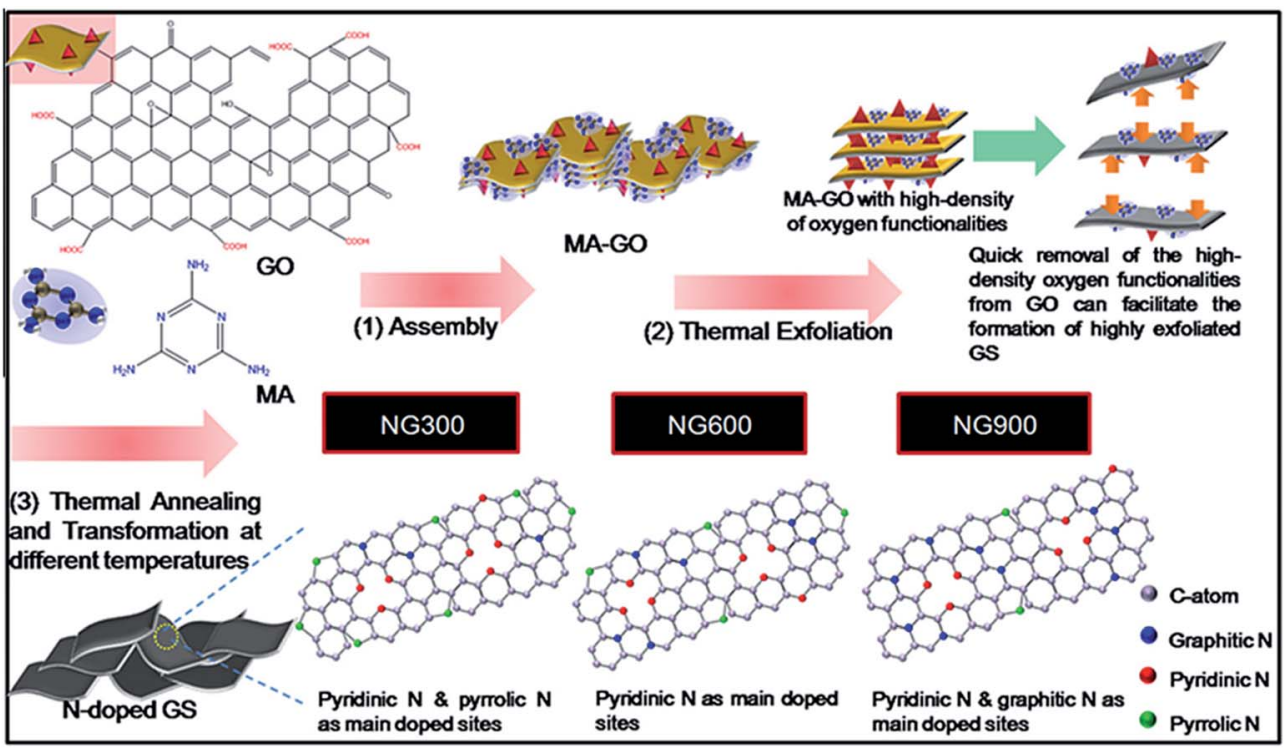

Fig. 10 Schematic illustration for the synthesis of $\mathrm{N}$-doped graphene with different allocation of nitrogen-doped configurations at different temperature. Reprinted with permission from ref. 88, Copyrights (2013) Elsevier Publications.

\subsection{Functionalized graphene as electrochemical sensor}

The unique and remarkable properties of graphene attracted tremendous interests in biosensor applications. However, the high specific area of graphene has a great tendency to reversibly aggregate and restack into graphite via $\pi-\pi$ stacking and van der Waals interactions. This further renders the superior features of graphene and limits the application. Functionalization or surface modification of graphene sheets by the attachment of functional groups via covalent or non-covalent methods onto graphene sheets can effectively prevent the agglomeration of single layer graphene by the presence of hydrophilic or hydrophobic group on graphene sheets. This facilitates the dispersion and homogeneity of graphene as well as maintaining the inherent properties of graphene. ${ }^{\mathbf{1 0 3 , 1 0 4}}$

In the case of functionalized graphene, Wu et al. ${ }^{105}$ reported a new type of modified electrode using porphyrin-functionalized graphene for the determination of DA. The negatively-charged porphyrin-functionalized graphene electrode stabilizes the chemically reduced graphene and thus facilitates the electron transfer rate at the electrode surface through the $\pi-\pi$ stacking interaction and electrostatic attraction whereas the suppression of the oxidation of AA and UA occurs to achieve a low detection limit of $0.01 \mu \mathrm{M}$. In another case, Li et al. ${ }^{\mathbf{1 0 6}}$ demonstrated a water-soluble sulfonated graphene (sulfonated-G) modified electrode for achieving improved selectivity and sensitivity for DA sensor in the existence of interference species (AA and UA). Similarly, this prepared anion sulfonated-G accelerates electron transfer reaction between the surface of electrode substrate and analytes via the $\pi-\pi$ stacking interaction and electrostatic attraction. Moreover, sulfonated-G offers better advancement over conventional Nafion film as the sulfonated-G is watersoluble which maintains the uniqueness and intriguing nature of graphene via electrostatic interactions.
Han and co-workers ${ }^{\mathbf{1 0 7}}$ has reported a ferulic acidfunctionalized electrochemically reduced graphene oxide (ERGO-FA) employed for the determination of DA in the existence of various interfering species (serotonin, glucose, $\mathrm{AA}, \mathrm{H}_{2} \mathrm{O}_{2}$, and UA). The prepared ERGO-FA/GCE exhibits good stability, increased selectivity, and sensitivity towards DA via amperometric responses in the DA concentration range of $0.6-1000 \mu \mathrm{M}$ with the LOD of $0.16 \mu \mathrm{M}(S / N=3)$. In a different study, Lian et al. ${ }^{108}$ utilized an ultrasonic route to synthesize tryptophanfunctionalized graphene (Trp-GR) through $\pi-\pi$ conjugate action between graphene and tryptophan (Fig. 11). This work reveals that the prepared Trp-GR have well dispersion behaviour in water and better conductivity than pure GR. The resulting Trp-GR/GCE modified electrode achieved excellent electrocatalytic performance for the simultaneous detection of DA, AA, and UA (Fig. 11).

Zhang et $a l .{ }^{109}$ successfully fabricated a simple, rapid, and selective sensing protocol using a tube-like structure graphene hybrid (GS-PTCA). The preparation of the resulting hybrid involved the combination between graphene sheets (GS) and 3,4,9,10-perylenetetracarboxylic acid (PTCA) via $\pi-\pi$ stacking interaction under ultrasonic condition. The proposed hybrid electrode offers improved catalytic activity and selectivity towards the oxidation of DA, AA, UA, and Trp as compared to the pure GS or PCTA. This can be attributed to the synergistic effects between GS and PCTA and also the unique structure of the modified layer PCTA that is enriched with carboxylic functional moieties attached on the surface of GS. In addition, this provides a selective interface to discriminate tracing analytes (DA, AA, UA, and Trp). Wang and co-workers ${ }^{\mathbf{1 1 0}}$ developed a modified electrode for the detection of electroactive species (DA, AA, and UA) using 1-butyl-3-methylimidazolium 2-amino-3mercaptopropionic acid salt ionic liquid functionalized graphene (IL-G/GCE). The superior electrochemical performance in simultaneous DA oxidation responses was mainly attributed 


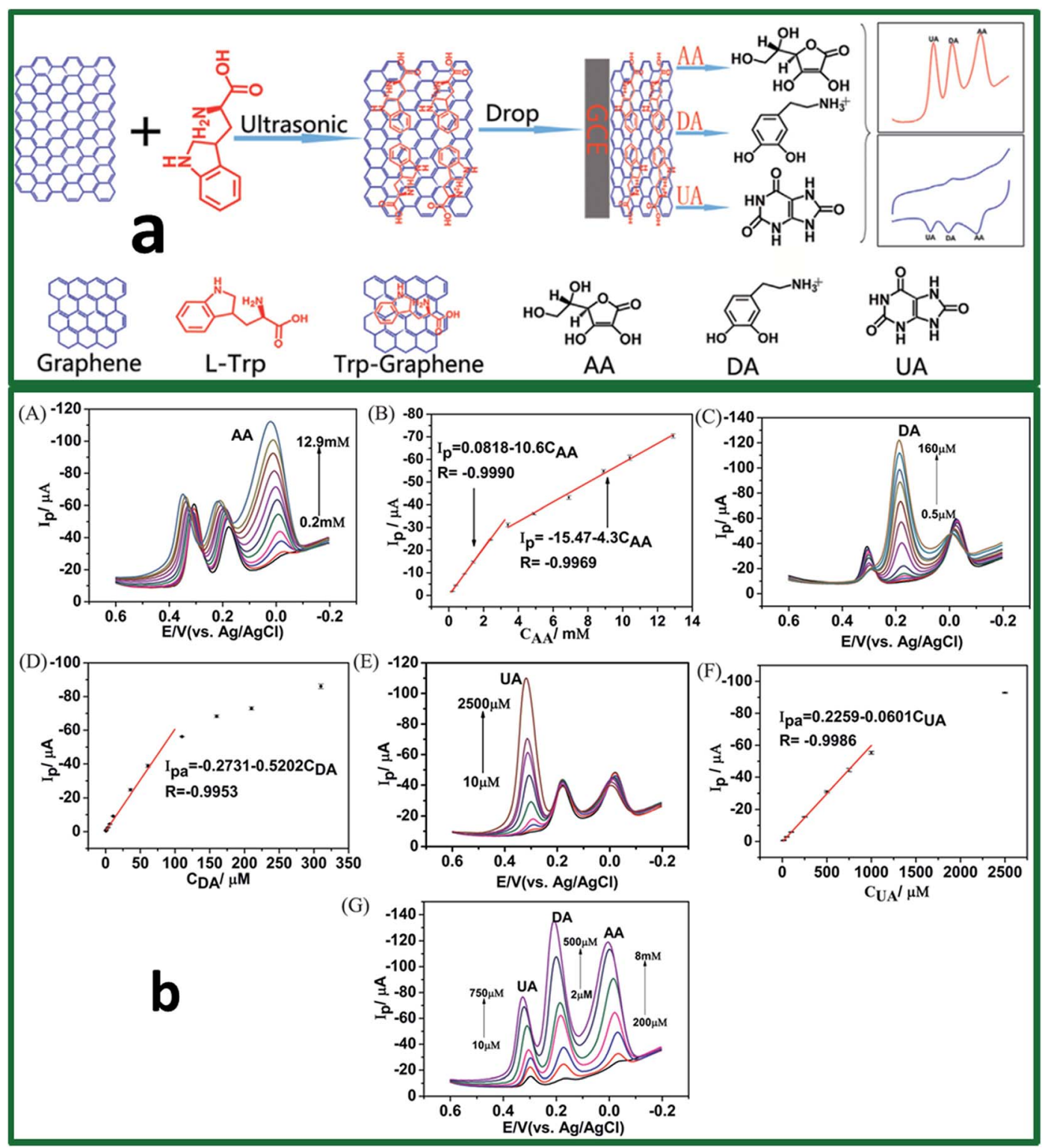

Fig. 11 (a) Schematic diagram of the fabrication procedure Trp-GR, Trp-GR/GCE, and the Trp-GR/GCE was used direct determination of AA, DA, and UA. (b) DPVs of AA, DA, and UA at Trp-GR/GCE in 0.1 M PBS (pH 7.0) respectively, scan rate: $50 \mathrm{mV} \mathrm{s}^{-1}$. (A) AA concentrations: 0.2, 0.4, 0.9, $1.4,2.4,3.4,4.9,6.9,8.9,10.4$, and $12.9 \mathrm{mM}$ in the presence of $50 \mathrm{mM}$ DA and $0.5 \mathrm{mM} \cup \mathrm{A}$; $(B)$ the linear relationship between currents and $A A$ concentration, (C) DA concentrations: $0.5,1.0,3.5,6.0,11,36,61,110,160,210$, and $310 \mathrm{mM}$ in the presence of $8 \mathrm{mM} \mathrm{AA}$ and $100 \mathrm{mM} \cup \mathrm{A}$; (D) the linear relationship between currents and DA concentration; (E) UA concentrations: 10, 20, 50, 100, 250, 500, 750, 1000, and 2500 mM in the presence of $50 \mathrm{mM}$ DA and $8 \mathrm{mM} \mathrm{AA}$; (F) the linear relationship between currents and UA concentration; (G) DPVs of different concentrations of AA, DA and UA $(0.2,0.45,0.95,2,4,6,8 \mathrm{mM})$ for AA, $(2,7,12,50,75,200$, and $500 \mathrm{mM})$ for DA and $(10,20,50,100,250,500$, and $750 \mathrm{mM})$ for $\mathrm{UA}$. Reprinted with permission from ref. 94, Copyrights (2014) Elsevier Publications.

to the synergistic effect between graphene and high ionic conductive IL.

Liu et al. ${ }^{\mathbf{1 1 1}}$ demonstrated a one pot preparation method for the graphene/ferrocene derivative (graphene/Fc- $\mathrm{NH}_{2}$ ) nanocomposite by mixing graphene and $\mathrm{Fc}-\mathrm{NH}_{2}$ solution. This nanocomposite was used to fabricate the Nafion/graphene/ $\mathrm{Fc}-\mathrm{NH}_{2}$ modified GCE for the detection of DA. The Fc- $\mathrm{NH}_{2}$ embedded on the graphene significantly enhances the charge-transport ability, stabilizes the graphene, and prevent the leakage of ferrocene. The Nafion/graphene/Fc- $\mathrm{NH}_{2} / \mathrm{GCE}$ significantly enhanced electrochemical response towards DA due to the synergistic amplification effect between the graphene and $\mathrm{Fc}-\mathrm{NH}_{2}$. The peak currents were linearly related to the DA concentrations in the range of $50 \mathrm{nM}$ to $200 \mu \mathrm{M}$ with the LOD of $20 \mathrm{nM}$. In the presence of $1 \mathrm{mM} \mathrm{AA}$ and $0.1 \mathrm{mM} \mathrm{UA}$, the electrode showed linear response in the range of 0.1 to $4 \mu \mathrm{M}$, and the detection limit was $50 \mathrm{nM}(S / N=3)$. Furthermore, this Nafion/Fc- $\mathrm{NH}_{2} /$ graphene/GCE was used for the detection of DA in serum and urine.

Mallesha and co-workers ${ }^{79}$ studied the electrochemical behaviour of octylamine-intercalated-graphene modified 
graphite electrode (FCGE) for the selective determination of DA in the presence of UA and AA. In their study, graphene was prepared via the solvothermal reduction of colloidal suspension of graphite oxide in which the FGCE showed excellent electrocatalytic activity towards DA, AA, and UA and successfully resolved the voltammetric signals of AA, DA, and UA into three distinct peaks as compared with that of the bare graphite electrode. Wang et al. ${ }^{\mathbf{1 1 2}}$ presented a method in preparing doped graphene into layered double hydroxide films (LDHs) for improving the charge transport of the LDH films for selectively sensing of DA. The high adsorption and catalytic activity of LDHs and superior electrical conductivity of graphene stimulates the strongly enhanced oxidative current response of DA, decreasing the oxidation over-potential as well as resolving the overlapped peak potentials of DA, AA, and UA into three distinct peaks. A summary of the various graphene, N-doped graphene, and functionalized graphene based electrochemical sensors for the detection of DA can be referred in Table 1. Among them, solution-gated graphene transistors (SGGTs) with functionalized gate electrodes showed the lowest LOD for DA (1 nM).

Determination of DA in real samples including urine and blood serum under optimal experimental conditions was performed to evaluate the applicability and feasibility of functionalized graphene modified electrodes in sensing applications. The obtained results are acceptable and satisfactory with the high recoveries of spiked samples ranging from 96.8 to $105 \%$ with R.S.D less than $3.0 \%$ for the detected samples, indicating that the modified electrode can be utilized for the simultaneous determination of DA in real human serum and pharmaceutical samples.

The highly sensitive and selective DA sensor based on TCPP/ CCG constructed by Wu et al. ${ }^{105}$ portrayed the best performance in DA oxidation among the reported functionalized graphene electrodes. Owing to the ion exchange membrane and graphene, the $\pi-\pi$ stacking and electrostatic interaction between positively charged DA molecules and negatively charged TCPP modified electrode are beneficial to promote the amount of DA molecules on the electrode and accelerate the electron transfer process on the surface of the electrode. Hence, the electrochemical sensor based functionalized graphene emerged as a good potential candidate in biosensing applications.

\subsection{Graphene-metal nanocomposite as electrochemical sensor}

Graphene-metal nanocomposite modified electrodes are one of the widely studied electrodes used in the detection of DA. The remarkable properties of metal nanoparticles incorporated on graphene depicts high electrocatalytic activity, excellent conductivity, and selectivity which makes metal nanostructures decorated on graphene an ideal choice to be used as an active material in electrochemical sensors. In addition, the absence of interfering oxidation and reduction peaks in graphene-metal nanocomposite in the DA sensors shows promising antiinterference effect.

Many researchers have investigated the usage of metal nanoparticles incorporated graphene nanocomposites modified electrode for the detection of DA. In terms of feasibility, noble metal particles in sub-10 $\mathrm{nm}$ dimensions are synthesized which gives this material the merit of higher surface area as compared to various metal oxide nanoparticles. The advantages of the electrochemical oxidation and reduction peaks of noble metals, is they do not overlap with the DA oxidation and reduction peaks that enable these metal nanoparticles to be used for DA sensing.

The gold nanoparticles (Au NPs) incorporated graphene sheets are one of the widely studied materials for biosensor. The significantly lower LOD value feature obtained towards the detection of DA by this graphene-Au NPs composite suggests that this material is suitable in the detection of the trace amount of DA. Li et al. ${ }^{113}$ reported the fabrication of graphene-Au NPs modified electrode by drop casting the preformed graphene and Au NPs solution on GCE surface. This fabricated graphene-Au NPs/GCE not only significantly improved the electrochemical peak potential separation between DA and AA, but also remarkably enhanced the current response and showed a wide linear concentration range of $5-1000 \mu \mathrm{M}$ with LOD of $1.86 \mu \mathrm{M}$ for DA. The graphene-Au NPs/GCE was stored in the refrigerator at $4{ }^{\circ} \mathrm{C}$ for two weeks in which the electrode showed the DPV current response of $0.1 \mathrm{mM}$ DA loss of only $5.7 \%$ in the initial response, indicating the good stability of the fabricated electrode. Further, the practical application of the electrode was demonstrated by determining DA in blood serum. Yang et $a l .{ }^{114}$ reported a sensitive, rapid, and accurate detection of DA in animal feed at low-cost for clinical diagnostic and therapeutic purposes with disposable ITO substrate embedded rGO-Au NPs. The ITO/rGO-Au NPs modified electrode was fabricated by direct reduction of GO on ITO and then Au NPs were electrochemically deposited by in situ on the ITO/rGO with fairly uniform density and size. The ITO/rGO$\mathrm{Au}$ NPs sensor electrode demonstrates the sensitivity of 62.7 $\mu \mathrm{A} \mathrm{mM}^{-1} \mathrm{~cm}^{-2}$ with good selectivity against most common electrochemically interfering species such as AA and UA for DA detection, and the LOD was $60 \mathrm{nM}(S / N=3)$ by DPV. The ITO/rGO-Au NPs sensor also exhibited good stability and capability to detect DA in beef samples whereby the LOD is consistent with optical sensor.

In another report, Liu and co-workers ${ }^{\mathbf{1 1 5}}$ demonstrated a simple, green, and controllable approach for electrochemical synthesis of a ERGO-Au nanocomposite made from ERGO and $\mathrm{Au}$ NPs for DA sensor. This nanocomposite possesses the specific features of both Au NPs and ERGO. The electrocatalytic studies concluded that the ERGO-Au NPs/GCE shows better electrocatalytic activity towards DA than bare GCE and its oxidation peak current is linearly proportional to the DA concentration in the range of $0.1-10 \mu \mathrm{M}$, with a LOD of $0.04 \mu \mathrm{M}$ $(S / N=3)$. Moreover, this ERGO-Au NPs/GCE showed good storage stability, reproducibility, and selectivity. Tian et al. ${ }^{\mathbf{1 1 6}}$ reported the synthesis of $\mathrm{Au}$ NPs $\beta$-cyclodextrin-graphene-(Au NPs- $\beta-C D-G r a)$ and modified GCE as a platform for DA sensing. Based on a SWV analysis, three well-resolved oxidation peaks of AA, DA, and UA were obtained for the Au NPs- $\beta-\mathrm{CD}-\mathrm{Gra} / \mathrm{GCE}$ and it exhibits good linear responses in the ranges 30-2000, 
Table 1 Comparison of various graphene, $\mathrm{N}$-doped graphene and functionalized graphene modified electrodes towards the detection of dopamine $^{a}$

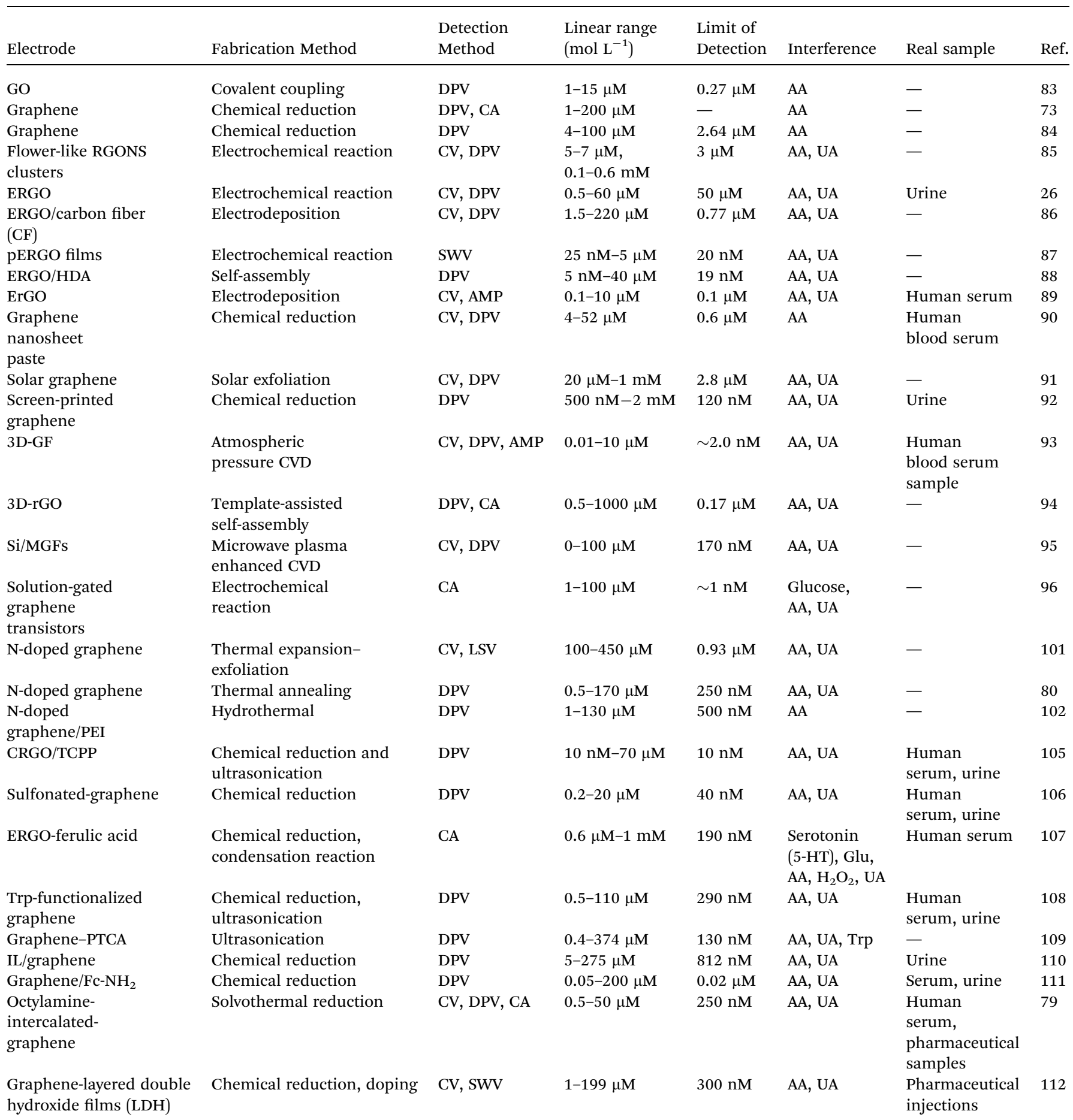

${ }^{a}$ ERGO: Electrochemically reduced graphene oxide; pERGO: partially electrochemically reduced graphene oxide; HDA: 1,6-hexanediamine; MGNFs: microwave plasma produced graphene nanoflake films; PEI: polyethylenimine; CRGO: chemically reduced graphene oxide; TCPP: meso-tetra(4carboxyphenyl)porphine; Glu: glucose; Trp: tryptophan; PTCA: 3,4,9,10-perylenetetracarboxylic acid; IL-G: 1-butyl-3-methylimidazolium 2-amino3-mercaptopropionic acid salt; LDH: layered double hydroxide films; CVD: chemical vapour deposition.

0.5-150 and 0.5-60 $\mu \mathrm{M}$, respectively. The LODs were found to be $10,0.15$, and $0.21 \mu \mathrm{M}(S / N=3$ and preconcentration time $=3$ min) for AA, DA, and UA, respectively.
Chen and co-workers ${ }^{\mathbf{1 1 7}}$ demonstrated their DA sensor using highly dispersed hollow gold-graphene nanocomposites (HAu-G) modified electrode. The $\mathrm{HAu}-\mathrm{G}$ were synthesized by a two-step 
method, involving the immobilization of hollow gold nanoparticles (HAu NPs) onto the surface of graphene sheets by mixing HAu NPs with a poly( $N$-vinylpyrrolidone)-functionalized graphene. The HAu-G/GCE electrode exhibits high electrocatalytic activity and extraordinary electronic transport properties. Amperometric detection of DA with this electrode showed a good linearity between 0.08 and $600 \mu \mathrm{M}$ with a LOD of $0.05 \mu \mathrm{M}$ $(S / N=3)$ and also demonstrates good reproducibility and operational stability. The interference of AA and UA was also excluded besides determining the DA in dopamine hydrochloride injection. Yan et al. ${ }^{118}$ demonstrated the layer-by-layer (LBL) assembled multilayer films of RGO/Au NPs as a sensor electrode for the DA sensor. A polysodium 4-styrenesulfonate (PSS)-RGO and polyamidoamine (PAMAM) dendrimer stabilized Au NPS uses the electrostatic LBL self-assembly on a GCE modified with a poly(diallyldimethylammonium chloride) (PDDA) as a primary layer. The electrochemical studies exhibit the LBL assembled RGO/Au NPs/GCE showing an excellent sensing performance for the detection of DA with LOD of $0.02 \mu \mathrm{M}(S / N=3)$ with a linear range from 1 to $60 \mu \mathrm{M}$. This sensor electrode further demonstrates the high sensitivity, selectivity, good analytical performance, and long-term stability due to the synergistic effect of RGO and Au NPs with in the LBL assembled films.

The Au NPs were used to decorate on the PEI wrapped graphene nanosheet (GNs) as reported by Ponnusamy et al. ${ }^{119}$ The mixture of graphene oxide nanosheets (GONS), PEI, and Au(III) undergoes a simultaneous microwave assisted chemical reduction to form GNS/PEI/Au NPs composite. The sodium borohydride $\left(\mathrm{NaBH}_{4}\right)$ was employed to further reduce the unreduced site of GONS and $\mathrm{HAuCl}_{4}$ in the PEI solution. This modified electrode gives a detection limit of $200 \mathrm{nM}$ with a sensitivity of $0.2 \mu \mathrm{A} \mu \mathrm{M}^{-1} \mathrm{~cm}^{-2}$. It is anticipated that an extensive research regarding the modification and development of new graphene-polymer based composite will be done in the future for improving the sensitivity of DA sensor. Liu et al. ${ }^{\mathbf{1 2 0}}$ reported a novel platform constructed for DA sensor based on RGO and Au NPS entrapped into the chitosan/silica sol-gel hybrid membranes (RGO-AuNPs-CSHMs). The hybrid membranes are prepared by cross-linking CS with APTES, while the presence of RGO and Au NPs that improves the conductivity of CSHMs. The RGO-AuNPs-CSHMs modified electrode exhibit enhanced sensitivity towards the oxidation of DA and UA and the CV and DPV techniques showed well defined and separated oxidation peaks for DA and UA with linear range of 1-200 $\mu \mathrm{M}$ and $1-300 \mu \mathrm{M}$, respectively. The LOD was found to be 0.3 and $0.7 \mu \mathrm{M}$ for DA and UA, respectively.

Qian et $a .^{\mathbf{1 2 1}}$ fabricated the Au NPs decorated PPy/RGO hybrid sheets for electrochemical DA sensor. The Au NPs-PPy/ RGO hybrid sheets prepared via an in situ chemical oxidative polymerization process, which showed crumpled and wrinkled surface of this composite along with the uniform flower-like $\mathrm{Au}$ nanoparticles and thus significantly improved the electrochemical properties of the modified electrode with surprisingly, an extremely low LOD of $18.92 \mathrm{pM}$ and a remarkable sensitivity of $16.40 \mu \mathrm{A} \mu \mathrm{M}^{-1}$ with a linear range of $0.1-5000 \mathrm{nM}$. Qian and co-workers used Au NPs coated polystyrene/reduced graphite oxide (PS/RGO) core shell microspheres via a facile process. ${ }^{122}$
They have mixed PS/RGO core shell microspheres and $\mathrm{AuCl}_{4}$ in aqueous solution by ultrasonication method before reducing the mixture together using trisodium citrate, $\mathrm{NaBH}_{4}$, and hydrazine hydrate via heat treatment. The Au NPs-PS/RGO microsphere modified electrode has shown an improvement in the sensibility of electrochemical detection for DA as low as 5 $\mathrm{nM}$ with a sensitivity of $3.44 \mu \mathrm{A} \mu \mathrm{M}^{-1}$ due to the unique chemical and electrical properties of citrate reduced Au NPs. Moreover, the coating of Au NPs also prevented the aggregation of RGO which then enhances the dispersibility and electrical conductivity of the composite.

Kaur et al. ${ }^{123}$ reported a simple synthetic method for the preparation of Ag NPs decorated rGO composite (Ag NPs/rGO), by heating the mixture of $\mathrm{GO}$ and $\mathrm{AgNO}_{3}$ in the presence of $\mathrm{NaOH}$. The Ag NPs/rGO was used to fabricate the sensor electrode for the simultaneous detection of UA, DA, AA, and Trp. The Ag NPs/rGO modified electrode exhibited excellent electrocatalytic activity, stability, sensitivity, and selectivity with well-separated oxidation peaks toward UA, DA, AA, and Trp. The higher electrocatalytic activity of $\mathrm{Ag} \mathrm{NPs} / \mathrm{rGO}$ is attributable to the facile transportation of electrons and reactant/product molecules due to the high surface area of GO and the uniform favorable distribution of $\mathrm{Ag}$ nanoparticles on rGO, providing a considerable surface enhancement effect to AA, DA, UA, and Trp, which significantly increases their oxidation current signals. The analytical performance of this $\mathrm{Ag}$ NPs/rGO composite material was demonstrated for the determination of AA and DA in commercial pharmaceutical samples such as vitamin $\mathrm{C}$ tablets and DA injections, respectively. Recently, Cincotto et al. ${ }^{124}$ demonstrated the preparation of $\sim 20 \mathrm{~nm}$ sized $\mathrm{Ag}$ NPs decorated mesoporous silica/graphene oxide $\left(\mathrm{SiO}_{2} / \mathrm{GO}\right)$ hybrid material by using DMF as a reducing agent. The Ag NPs/ $\mathrm{SiO}_{2} / \mathrm{GO}$ hybrid material was used to develop a sensitive electrochemical sensor for the simultaneous determination of epinephrine (Ep) and DA. The study demonstrates that the resultant $\mathrm{Ag} \mathrm{NPs} / \mathrm{SiO}_{2} / \mathrm{GO}$-modified electrode is highly sensitive for the simultaneous determination of DA and Ep with the LOD of 0.26 and $0.27 \mu \mathrm{M}$, respectively. The improved selectivity and sensitivity of this modified electrode is due to the synergistic effect characteristic of $\mathrm{Ag} \mathrm{NPs}, \mathrm{SiO}_{2}$, and $\mathrm{GO}$ in the hybrid material. The $\mathrm{Ag} \mathrm{NPs} / \mathrm{SiO}_{2} / \mathrm{GO}$-modified electrode is highly selective and was used to detect the DA and Ep in human urine samples.

$\mathrm{Xu}$ et al. ${ }^{125}$ demonstrated a simultaneous electrochemical detection of DA, UA, and AA using Pt/reduced graphene oxide (Pt/RGO) modified GCE. The Pt/RGO nanocomposite was prepared by the chemical reduction of $\mathrm{H}_{2} \mathrm{PtCl}_{6}$ and GO, and was used for electrode modification. The Pt/RGO/GCE modified electrode showed good performance towards the individual and also simultaneous detection of DA and UA in the presence of 1 mM AA. The oxidation peak currents showed linear relationship with the associated DA and UA concentrations in the range of 10-170 $\mu \mathrm{M}$ and 10-130 $\mu \mathrm{M}$, respectively, with the LOD of 0.25 $\mu \mathrm{M}$ for DA and $0.45 \mu \mathrm{M}$ for UA $(S / N=3)$. The electrode showed good reproducibility and the RSDs of the peak currents of 30 successive runs are 3.75 and $3.22 \%$ for DA and UA, respectively, which are both lower than $4 \%$, and it suggest that the Pt/RGO/ 
GCE is not subjected to surface fouling effect by the oxidized products. Zhi-Quan and co-workers ${ }^{\mathbf{1 2 6}}$ reported the graphenepoly(styrene sulfonate)-Pt nanocomposite (GN-PSS-Pt) based DA sensor. A clean and facile microwave-heating procedure was used for the preparation of well-dispersed GN-PSS-Pt nanocomposite by using microwave-heating method. The GO and chloroplatinic acid could be simultaneously reduced with ethylene glycol and used for electrochemical study. In this study, a chronoamperometric technique was used for the DA detection and the electrode showed the linear range of $200 \mathrm{nM}$ to $4 \mathrm{mM}$ with the LOD of $40 \mathrm{nM}(S / N=3)$. The GN-PSS-Pt/GCE showed good repeatability, even when the electrode was subjected to the oxidation of $300 \mu \mathrm{M}$ of DA for 11 times which stills exhibit a satisfying repeatability with a RSD of $4.3 \%$. The response of the electrode for $300 \mu \mathrm{M}$ DA was $92.5 \%$ of its initial current response after storage for 10 days. The modified electrodes exhibit good selectivity toward DA sensing in the presence of interfering species $\mathrm{Ca}^{2+}, \mathrm{K}^{+}, \mathrm{Na}^{+}, \mathrm{Zn}^{2+}, \mathrm{Cl}^{-}, \mathrm{Mg}^{2+}$, $\mathrm{CH}_{3} \mathrm{COO}^{-}, \mathrm{SO}_{4}{ }^{2-}, \mathrm{H}_{2} \mathrm{PO}_{4}{ }^{-}, \mathrm{HPO}_{4}{ }^{2-}$, UA, and AA. Moreover, the GN-PSS-Pt modified electrode was applied for the determination of DA in human urine and serum samples.

Li et $a l .{ }^{74}$ reported a one pot preparation method to prepare a water-soluble and electroactive composite, Pt NPs/ polyelectrolyte-functionalized ionic liquid/graphene sheets (Pt/ PFIL/GS) by chemical reduction. The obtained Pt/PFIL/GS nanocomposite was used to fabricate the modified electrode for the simultaneous detection of AA and DA. The difference between the oxidation potentials of AA and DA is over $200 \mathrm{mV}$, which leads to the ability to distinguish AA from DA. The obtained potential difference is due to $\pi-\pi$ interactions between the phenyl structure of DA and planar hexagonal carbon structure of graphene which facilitates the easy arrival of DA molecules to the surface of modified electrode. Although the $\pi-\pi$ interaction between penta-heterocycle of AA and planar hexagonal carbon structure of graphene is weak, the presence of cationic PFIL provides a remarkable electrostatic attraction to the negatively charged AA and rejection to positively charged DA in PBS ( $\mathrm{pH} 7.0)$ due to the charged characteristic of $\mathrm{AA}\left(\mathrm{p} K_{\mathrm{a}}=\right.$ 4.10) and DA ( $\left.\mathrm{p} K_{\mathrm{a}}=8.87\right)$. The electrostatic force leads to the realization of $\mathrm{AA}$ oxidation at the $\mathrm{Pt} / \mathrm{PFIL} / \mathrm{GS}$-modified electrode. The sensor electrode was further used for real sample analysis (human urine), and it showed independent oxidation peaks for urine sample containing AA and DA. In another report, ${ }^{127}$ size-selected Pt nanoparticles with a mean diameter of $1.7 \mathrm{~nm}$ were self-assembled onto the graphene surface and was used for the simultaneous electrochemical detection of AA, DA, and UA. Based on the DPV technique, DA in the presence of UA and AA depicts three isolated peak separation potentials were $185 \mathrm{mV}$ (AA to DA), $144 \mathrm{mV}$ (DA to UA), and $329 \mathrm{mV}$ (AA and UA), respectively, indicating the good selectivity of the Pt/graphene modified electrode.

Chen and co-workers ${ }^{128}$ studied the electrochemical sensing behaviour of palladium-electrochemically reduced graphene oxide (Pd NPs/RGO) modified electrode towards DA. The Pd NPS/RGO modified electrode showed a DA oxidation peak at $0.25 \mathrm{~V}$. Upon increasing the concentration of DA, the oxidation peak current increased linearly, but the reduction peak current decreased which is due to the high surface area and high conductivity of Pd NPs/RGO nanocomposite. Further, it showed a linear concentration range of $1-150 \mu \mathrm{M}$ and LOD of $0.233 \mu \mathrm{M}$ $(S / N=3)$ with a sensitivity of $2.62 \mu \mathrm{A} \mu \mathrm{M}^{-1} \mathrm{~cm}^{2}$. Instead of using ERGO, another study reported a chemically reduced graphene oxide (CRGO) sheets which was used in the nanocomposite for the DA sensor. ${ }^{129}$ The presence of CRGO in the Pd NPs/CRGO/ GCE electrode enhances the surface coverage concentration and also increases the electron transfer rate constant. The Pd NPs/CRGO/GCE showed two well separated oxidation peaks for the detection of DA and diclofenac in a mixture with a linear range of 2-63 $\mu \mathrm{M}$ and was further used for the simultaneous detection of DA and diclofenac in real samples with a linear range of 2-50 $\mu \mathrm{M}$. Wu et al. ${ }^{130}$ reported a high-performance nonenzymatic electrochemical sensor using high-conductivity and large-surface-area graphene supported Pd NPs in the presence of supercritical $\mathrm{CO}_{2}$ fluid $\left(\mathrm{scCO}_{2}\right)$. The merit of using the $\mathrm{scCO}_{2}$ is that they can de-bundle the graphene nanosheets and uniformly disperse the Pd NPs $(\sim 3 \mathrm{~nm})$, thereby effectively suppressing the restacking of graphene. The Pd NPs are sandwiched between the highly conductive sheets leading to superior electrochemical utilization. Consequently, the $\mathrm{Pd} /$ graphene@scCO $\mathrm{CO}_{2}$ exhibited significantly higher sensitivity and selectivity for AA, DA, and UA as compared to those of the plain graphene and Pd/graphene@air electrodes. The electrode showed sensitivity of $287 \mu \mathrm{A} \mu \mathrm{M}^{-1} \mathrm{~cm}^{2}$ and LOD of $0.1 \mu \mathrm{M}$ $(S / N=3)$ for DA with co-existence of $1 \mathrm{mM}$ AA and $50 \mu \mathrm{M}$ UA.

Jiang and co-workers ${ }^{\mathbf{1 3 1}}$ described the one-step in situ reduction process for the preparation of bimetallic $\mathrm{Pd}-\mathrm{Pt}$ nanoparticles anchored on functionalized reduced graphene oxide (RGO) nanocomposite, in which Pt and Pd ions were firstly attached to PDDA-functionalized graphene oxide (GO) sheets and then the encased metal ions and GO were subjected to simultaneous reduction by ethylene glycol. An electrochemical sensor based on the $\mathrm{Pd}_{3} \mathrm{Pt}_{1} / \mathrm{PDDA}-\mathrm{RGO}$ nanocomposites was fabricated for the simultaneous detection of AA, DA, and UA in their ternary mixture and it showed three wellseparated voltammetric peaks along with remarkable increasing electro-oxidation currents by DPV technique. Under the optimized conditions, the linear relationships between the peak currents and the concentrations were in the range of 40$1200 \mu \mathrm{M}$ (AA), 4-200 $\mu \mathrm{M}$ (DA), and 4-400 $\mu \mathrm{M}$ (UA) with LOD $(S / N=3)$ of $0.61,0.04$, and $0.10 \mu \mathrm{M}$ for $\mathrm{AA}, \mathrm{DA}$, and $\mathrm{UA}$, respectively. The improved sensing performance can be attributed to the synergistic effect of Pd-Pt bimetallic nanoparticles and RGO. Further, the practical utility of the sensor electrode was demonstrated by the determination of AA, DA, and UA in human urine and blood serum samples.

Recently, Jiang and co-workers ${ }^{\mathbf{1 3 2}}$ have reported reduced graphene oxide-supported Au@Pd (Au@Pd-;RGO) nanocomposites for the simultaneous determination of AA, DA, and UA (Fig. 12). The fabricated Au@Pd-;RGO electrodes show good sensing properties due to their large surface area and the conductivity of RGO. Hence, the linear response ranges were 1$800 \mu \mathrm{M}, 0.1-100 \mu \mathrm{M}$, and $0.1-350 \mu \mathrm{M}$ with the LOD of 0.28 , 0.024 , and $0.02 \mu \mathrm{M}$ for AA, DA, and UA, respectively. A summary of the various graphene-metal nanocomposites based 


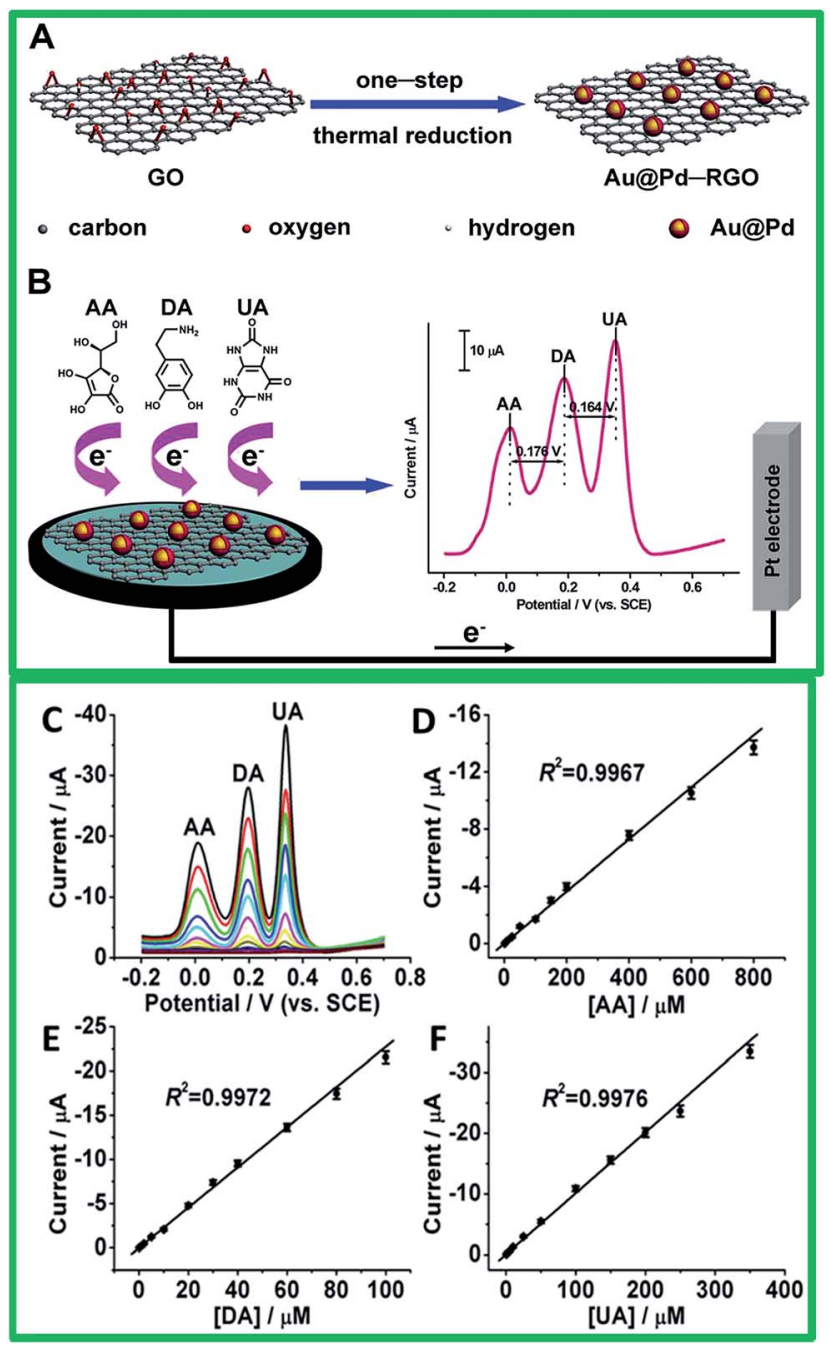

Fig. 12 Schematic Illustration of preparations of (A) Au@Pd-RGO nanocomposites and (B) AuQPd-RGO/GCE sensors for simultaneous determination of AA, DA, and UA. (C and E) From bottom to up 60 the concentrations of AA from 1 to $800 \mu \mathrm{M}$, DA from 0.1 to $100 \mu \mathrm{M}$, and UA from 0.1 to $350 \mu \mathrm{M}$. ( $D$ and $F$ ) Plots of the oxidation peak currents against the concentrations of AA, DA, and UA, respectively. The error bars represent standard deviations for five tests. Reprinted with permission from ref. 120, Copyrights (2014) Royal Society of Chemistry.

electrochemical sensor in the detection of DA can be referred in Table 2.

In real sample analysis involving blood serum and urine, few reported works involving the use of GN-PSS-Pt, graphene/Pd$\mathrm{Pt}, \mathrm{Au} / \mathrm{graphene}$, AuNPs/PS/RGO microspheres, and Au/PPy/ RGO to detect DA with high amount of recovery. ${ }^{113,121,122,126}$ Similarly, other graphene-metal nanocomposites also showed applicability in the detection of DA in real conditions. Among the reported graphene-metal nanocomposites, the Au@PPy/ RGO modified electrode reported by T. Qian et al. ${ }^{108}$ is the most promising choice because they have achieved the lowest LOD. This nanocomposite features offer the formation of flower-like $\mathrm{Au}$ nanoparticles into the PPy/RGO which exhibits a unique structure, thus enhancing the electrical conductivity that leads to the excellent electrocatalytic activity towards DA.

\subsection{Graphene-metal oxide nanocomposite as electrochemical sensor}

A small scale of research works using graphene-metal oxide based nanocomposites for the sensing of DA has been established in recent years. Although graphene-metal based nanocomposites were investigated widely in its enhanced composite features for the detection of DA, other alternative cost-effective materials were still explored to replace metal nanoparticles. Thus, this leads to metal oxides being studied in accomplishing this target since it is high in abundance, cost-effective, and "green". The addition of these metal oxide nanostructures on graphene forms composite materials that reveals promotional benefits in improving electrochemical properties which cannot be achieved by either component alone. The use of graphenemetal oxide nanocomposites in detecting DA was first reported in 2010 by Fan et al. ${ }^{75}$ using $\mathrm{TiO}_{2}$ /graphene nanocomposite based on a simple reaction of graphene and titanium isopropoxide through hydrothermal process. Their experiments have shown a good LOD $(2 \mu \mathrm{M})$ and the selective nature of the nanocomposite modified on GCE in detecting DA under the interference effect of AA and UA. In a related study, another report reveals the use of electropolymerization of 4-aminobenzenesulfonic acid (4-ABSA) on modified graphene/titanium dioxide $\left(\mathrm{TiO}_{2}-\mathrm{GR}\right) \mathrm{GCE}$ which exhibits fast electron transfer

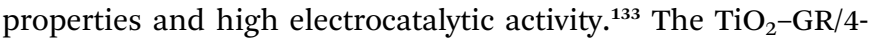
ABAS/GCE shows a good LOD of $0.1 \mu \mathrm{M}$ and the ability to simultaneously detect DA and tryptophan (Trp), even under various interference species (AA, glucose, vitamin B6, tyrosine and cysteine). We have also previously reported a reduced graphene oxide/titanium dioxide nanocomposite with highly exposed facets $\left(\mathrm{rGO} / \mathrm{TiO}_{2}\{001\}\right)$ through a simple solvothermal reaction with diethlyenetriamine as the facet growth manipulating agent. ${ }^{134}$ The modified $\mathrm{rGO} / \mathrm{TiO}_{2} \quad\{001\}$ GCE shows enhanced electrochemical sensing towards DA under the interference effect of AA that certainly opens up a new platform of facet manipulation studies for electrochemical applications (Fig. 13).

Another graphene-metal oxide nanocomposite that utilizes the electrical conductive and superparamagnetism properties of $\mathrm{Fe}_{3} \mathrm{O}_{4}$ on reduced graphene oxide sheets $\left(\mathrm{Fe}_{3} \mathrm{O}_{4} / \mathrm{r}-\mathrm{GO}\right)$ was reported by Teymourian et al. ${ }^{135} \mathrm{~A}$ simple chemical reduction method using hydrazine hydrate to form $\mathrm{Fe}_{3} \mathrm{O}_{4} / \mathrm{r}$-GO proves superior in the enhancement of bioelectrochemical sensing of various analytes such as $\mathrm{NADH}$, lactate, $\mathrm{O}_{2}$, and $\mathrm{H}_{2} \mathrm{O}_{2}$. In the context of DA, their modified GCE shows clear separation of peak potentials of DA, AA, and UA with LOD of 0.08, 20.0, and $0.50 \mu \mathrm{M}$, respectively. However, the use of hydrazine and hydrazine hydrate compounds are not favourable in terms of their high toxicity and instability. Another nanocomposite based on $\mathrm{Fe}_{3} \mathrm{O}_{4}$ was also reported using amino-group functionalized mesoporous $\mathrm{Fe}_{3} \mathrm{O}_{4}$ on graphene sheets $\left(\mathrm{Fe}_{3} \mathrm{O}_{4}-\mathrm{NH}_{2} @ G S\right) .{ }^{136}$ Their $\mathrm{Fe}_{3} \mathrm{O}_{4}-\mathrm{NH}_{2} @ \mathrm{GS} / \mathrm{GCE}$ shows high sensitivity and selectivity towards simultaneous detection of DA, AA, and UA. Hence, this nanocomposite offers high biocompatibility and good electrocatalytic oxidation which is simple and environmental friendly to produce. Salamon et al. ${ }^{137}$ 
Table 2 Comparison of various graphene-metal nanocomposite modified electrodes towards the detection of dopamine ${ }^{a}$

\begin{tabular}{|c|c|c|c|c|c|c|c|}
\hline Electrode & Fabrication method & $\begin{array}{l}\text { Detection } \\
\text { method }\end{array}$ & Linear range & $\begin{array}{l}\text { Limit of } \\
\text { detection }\end{array}$ & Interference & Real sample & Ref. \\
\hline $\mathrm{Au}$-graphene & Chemical reduction & DPV & $5-1000 \mu \mathrm{M}$ & $1.86 \mu \mathrm{M}$ & $\mathrm{DA}, \mathrm{AA}$ & Human serum & 113 \\
\hline ERGO-Au NPs & $\begin{array}{l}\text { Electrochemical reduction } \\
\text { and deposition }\end{array}$ & & $0.1-10 \mu \mathrm{M}$ & $0.04 \mu \mathrm{M}$ & $\begin{array}{l}\mathrm{NaCl}, \mathrm{KNO}_{3}, \\
\mathrm{MgSO}_{4}, \mathrm{UA}, \\
\mathrm{AA}, \mathrm{Glu}, \mathrm{Cys}, \\
\mathrm{CA}, \mathrm{TA}, \mathrm{Lys}\end{array}$ & $\begin{array}{l}\text { Dopamine } \\
\text { hydrochloride } \\
\text { injection, urine }\end{array}$ & 115 \\
\hline HAu-graphene & Chemical reduction & DPV & $0.08-600 \mu \mathrm{M}$ & $50 \mathrm{nM}$ & DA, UA, AA & - & 117 \\
\hline Au@PSS-graphene & Chemical reduction & DPV & $1-60 \mu \mathrm{M}$ & $20 \mathrm{nM}$ & $\mathrm{DA}, \mathrm{UA}, \mathrm{AA}$ & - & 118 \\
\hline $\mathrm{G} / \mathrm{PEI} / \mathrm{Au}$ & Chemical reduction & DVP & $2-48 \mu \mathrm{M}$ & $200 \mathrm{nM}$ & $\mathrm{DA}, \mathrm{AA}$ & Urine & 119 \\
\hline RGO-AuNPs-CSHMs & Chemical reduction & DPV & $0-200 \mu \mathrm{M}$ & $50 \mathrm{nM}$ & $\begin{array}{l}\text { DA, UA, AA, } \\
\text { Glu }\end{array}$ & Urine & 120 \\
\hline Au@PPy/RGO & Chemical reduction & DPV & $0-5 \mu \mathrm{M}$ & $18.92 \mathrm{pM}$ & $\mathrm{DA}, \mathrm{UA}, \mathrm{AA}$ & $\begin{array}{l}\text { Urine, human } \\
\text { serum }\end{array}$ & 121 \\
\hline $\mathrm{Ag} \mathrm{NPs} / \mathrm{SiO}_{2} / \mathrm{GO}$ & In situ chemical reduction & & $2-80 \mu \mathrm{M}$ & $0.26 \mu \mathrm{M}$ & UA, AA, EA & Urine & 124 \\
\hline $\mathrm{Pt} / \mathrm{RGO}$ & Chemical reduction & DPV & $10-170 \mu \mathrm{M}$ & $250 \mathrm{nM}$ & $\mathrm{DA}, \mathrm{UA}, \mathrm{AA}$ & - & 125 \\
\hline GN-PSS-Pt & Microwave reduction & CA & $20 \mathrm{nM}-4 \mathrm{mM}$ & $40 \mathrm{nM}$ & $\begin{array}{l}\mathrm{Ca}^{2+}, \mathrm{K}^{+}, \mathrm{Na}^{+}, \\
\mathrm{Zn}^{2+}, \mathrm{Cl}^{-}, \mathrm{Mg}^{2+}, \\
\mathrm{CH}_{3} \mathrm{COO}^{-}, \mathrm{SO}_{4}{ }^{2-}, \\
\mathrm{H}_{2} \mathrm{PO}_{4}{ }^{-}, \mathrm{HPO}_{4}{ }^{2-}, \\
\mathrm{UA}, \mathrm{AA}\end{array}$ & $\begin{array}{l}\text { Urine, human } \\
\text { serum }\end{array}$ & 126 \\
\hline Pt/PFIL/GS & Chemical reduction & DPV & - & - & $\mathrm{DA}, \mathrm{UA}, \mathrm{AA}$ & Urine & 74 \\
\hline Graphene/Pt & Reflux & DPV & $0.03-8.13 \mu \mathrm{M}$ & $30 \mathrm{nM}$ & $\mathrm{DA}, \mathrm{UA}, \mathrm{AA}$ & - & 127 \\
\hline RGO-Pd & Electrochemical reduction & LSV & $1-150 \mu \mathrm{M}$ & $233 \mathrm{nM}$ & DA, UA, AA, Glu & - & 128 \\
\hline
\end{tabular}

${ }^{a} \mathrm{Au}$ : gold nanoparticles; $\beta$-CD: $\beta$-cyclodextrin; ERGO: electrochemically reduced graphene oxide; RGO: reduced graphene oxide; PSS: polysodium 4styrenesulfonate; PEI: polyethylenimine; CSHMs: chitosan/silica sol-gel hybrid membranes; Ag: silver nanoparticles; Pt: platinum nanoparticles; PFIL: polyelectrolyte-functionalized ionic liquid; Pd: palladium nanoparticles; PDDA: poly(diallyldimethylammonium chloride).

reported the magnetite nanorods anchored over rGO synthesized through a simple and time efficient one pot method $(\mathrm{rGO} /$ $\mathrm{Fe}_{3} \mathrm{O}_{4}$ ). Interestingly, the LOD for the $\mathrm{rGO} / \mathrm{Fe}_{3} \mathrm{O}_{4}$ composite achieved a value of $7 \mathrm{nM}$ towards the detection of DA owing to the large surface area of GO which provides high electrical conductivity, biocompatibility, and aromatic $\mathrm{sp}^{2}$ bonds for the facile transportation of electrons. In addition, the cubic spinel structure of the $\mathrm{Fe}_{3} \mathrm{O}_{4}$ nanorods also increases adsorption therefore enhancing electrocatalytic activity. We have also reported an iron oxide/graphene modified glassy carbon electrode $\left(\mathrm{Fe}_{3} \mathrm{O}_{4} / \mathrm{rGO} / \mathrm{GCE}\right)$ and its simultaneous detection of DA and $\mathrm{AA}$, in which the $\mathrm{Fe}_{3} \mathrm{O}_{4} / \mathrm{rGO}$ was prepared through a one step in situ wet chemical method. ${ }^{138}$ Through this, the detection limit of $\mathrm{Fe}_{3} \mathrm{O}_{4} / \mathrm{rGO} / \mathrm{GCE}$ was found to be 0.42 and $0.12 \mu \mathrm{M}$ for AA and DA in the concentration range of 1-9 $\mathrm{mM}$ and $0.5-100 \mu \mathrm{M}$ showing the possible use of $\mathrm{Fe}_{3} \mathrm{O}_{4} / \mathrm{rGO}$ as a potential electrode material.
Tin dioxide on graphene sheets used in modifying carbon ionic liquid electrode $\left(\mathrm{GR}-\mathrm{SnO}_{2} / \mathrm{CILE}\right)$ was first introduced by Sun et $a l .{ }^{139}$ for electrochemical sensing application. They reported the detection of DA with a detection limit of $0.13 \mu \mathrm{M}$ even under high dosage of UA as the interference compound. Moreover, the modified electrode exhibits excellent recovery of DA in the injection samples. Similarly, another report was described on graphene oxide (GO) functionalized with sodium dodecyl sulphate to synthesize graphene nanosheet/ $\mathrm{SnO}_{2}$ nanoparticle hybrid nanocomposite (SDS-GN/SnO ${ }_{2}$ ) ${ }^{140}$ The use of SDS in the nanocomposite prevents the restacking of graphene layers and enhanced the electrochemical properties in which the SDS-GN/SnO $/$ GCE portray a LOD of $80 \mathrm{nM}$ in determining DA and also high recovery of DA in human urine tests. However, the use of hydrazine hydrate in their synthesis method still remains a negative impact.

Other graphene-metal oxide nanocomposite studies includes the use of cuprous oxide decorated on graphene sheets 

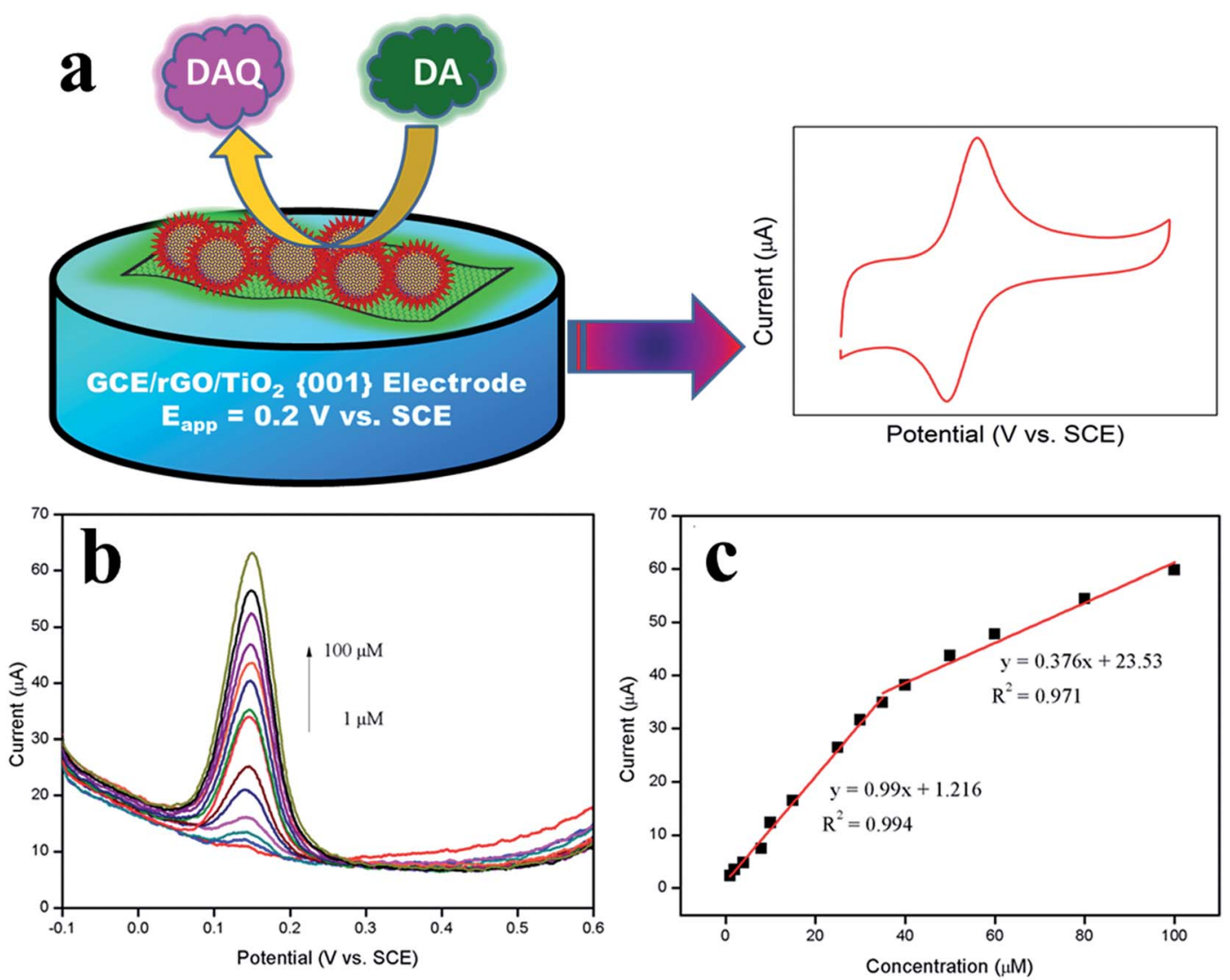

Fig. 13 (a) Mechanism for the electrocatalytic oxidation of dopamine at $\mathrm{rGO} / \mathrm{TiO}_{2}\{001\}$ modified GCE. (b) DPV obtained for $\mathrm{rGO} / \mathrm{TiO} 2$ \{001\} modified GCEs during the addition of $1,2,4,8,10,15,25,30,35,40,50,60,80$, and $100 \mu$ M DA into the $0.1 \mathrm{M} \mathrm{PBS}$ (pH=6.5). (c) Calibration plot observed for the oxidation peak current vs. concentration of DA. Reprinted with permission from ref. 123, Copyrights (2014) Nature publishing Group.

$\left(\mathrm{Cu}_{2} \mathrm{O} /\right.$ graphene $)$ reported by Zhang et al. ${ }^{141}$ Their nanocomposite offers uniform $\mathrm{Cu}_{2} \mathrm{O}$ nanoparticles broadly distributed on graphene sheets through a solvothermal route in ethylene glycol. Based on their $\mathrm{CV}$ studies, their $\mathrm{Cu}_{2} \mathrm{O} /$ graphene/GCE denotes a good LOD of $10 \mathrm{nM}$ in detecting DA but increases to $200 \mathrm{nM}$ as UA was added as the interference effect with the selectivity properties still satisfactory. As mentioned above, most of the studies employ graphene as their platform to grow metal oxides nanostructures. Nonetheless, an investigation was reported in using $\mathrm{SiO}_{2}$ coated GO instead as a surface to prepare molecularly imprinted polymers (GO/SiO 2 -MIPs) composites. ${ }^{142}$ This interesting method prepares $\mathrm{GO} / \mathrm{SiO}_{2}$ through sol-gel process and the introduction of vinyl groups onto $\mathrm{GO} / \mathrm{SiO}_{2}$ with $\gamma$-methacryloxypropyltrimethoxysilane ( $\gamma$-MAPS) and finally copolymerization. Indeed, their fabricated $\mathrm{GO} / \mathrm{SiO}_{2}-\mathrm{MIPS} / \mathrm{GCE}$ shows promising electrochemical sensing properties and excellent selective characteristics of DA over AA, UA, norepinephrine (NE), and epinephrine (EP). Ma et al. ${ }^{143}$ reported a three-dimensional graphene (3DGN)@WO $\mathrm{W}_{3}$ nanowire sensor in detecting $\mathrm{H}_{2} \mathrm{O}_{2}$, $\mathrm{AA}$, and DA. Their unique structure shows promising results in high electrocatalytic activities which have the potential to act as a scaffold support and as a real time sensor monitoring analytes. Lastly, Nayak et al. ${ }^{144}$ reported a Nafion coated $\mathrm{ZnO}$ nanoparticles decorated graphene sheets (ZnO-sG-Nafion) as a transducer candidate. Their fabricated modified electrode shows a good linear amperometric response from $10 \mathrm{M}$ to $800 \mathrm{M}$ with a LOD of $1 \mathrm{M}$. A summary of the various graphene-metal oxide nanocomposites based electrochemical sensor in the detection of DA can be referred in Table 3.

The real sample analysis was carried out in few reported works involving human serum and urine and the recovery of DA achieved 90 to $110 \%$, indicating the potential use of graphenemetal oxide nanocomposite to modify electrochemical sensor for practical application. Among the reported metal oxides incorporated graphene nanocomposites, the $\mathrm{rGO} / \mathrm{Fe}_{3} \mathrm{O}_{4}$ modified electrode have the lowest LOD of $7 \mathrm{nM}$ for DA detection which also indicates good reproducibility $(\mathrm{RSD}=2.85 \%)$ and remarkable anti-interference effect when AA and UA was added into the DA based solution. ${ }^{137}$ This fabrication method is quite promising in applying the graphene-metal oxide nanocomposite unique features as it involves a one-pot preparation route without the addition of surfactants making it an ideal choice. Based on all of this, there is still room for more investigation on graphene-metal oxide based nanocomposites for electrochemical sensing of DA as this field is still not widely explored. Hopefully, future works would hold promising results 
Table 3 Comparison of various graphene-metal oxide nanocomposite modified electrodes towards the detection of dopamine ${ }^{a}$

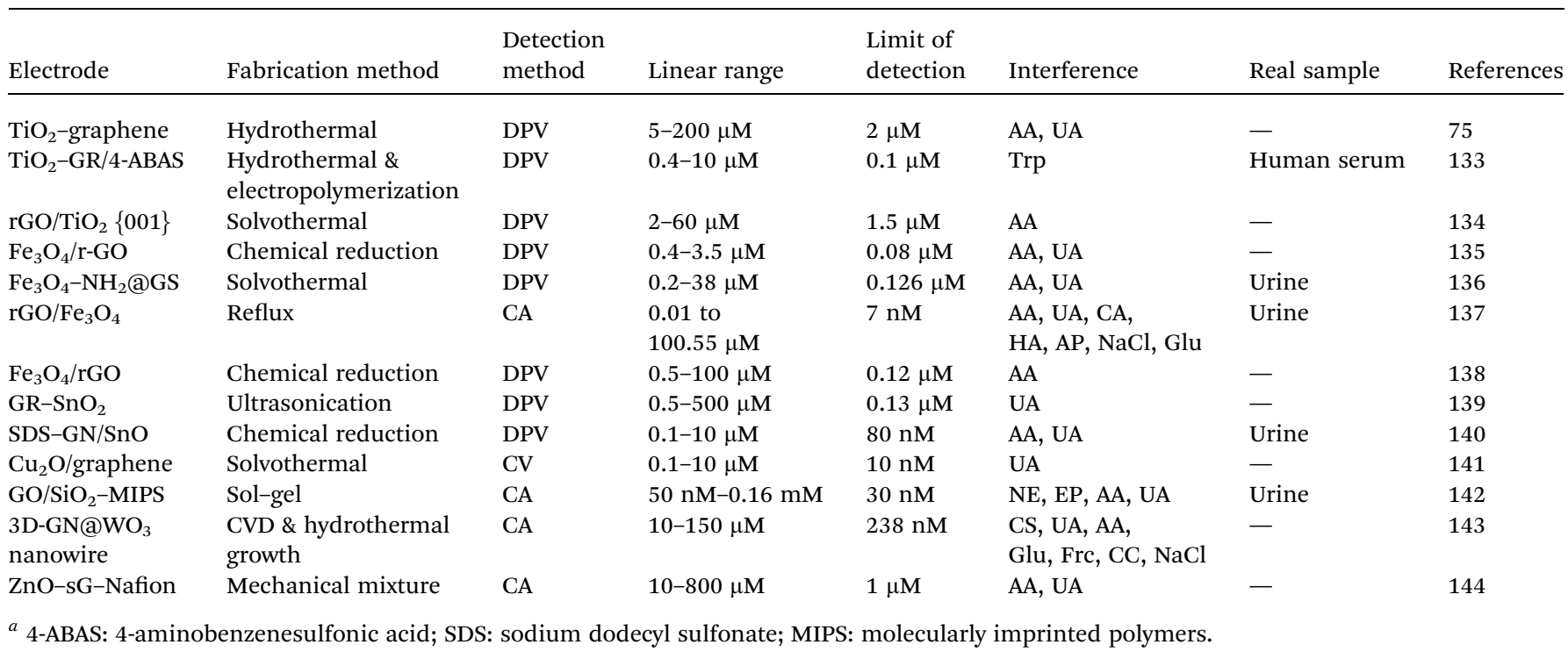

in the key of producing cheaper and greener composite materials for electrochemical sensing applications.

\subsection{Graphene-polymer composite as electrochemical sensor}

Currently, the research on graphene has moved one step forward when (where) the researchers has successfully proved the ability of graphene as a strong and conductive filler for polymer matrix to form graphene based polymer composites. The high surface area and outstanding thermal and electrical conductivity properties possessed by graphene enables it to be the most suitable candidate as filler for polymer matrix. In addition, the precursor for graphene (which is graphite) is also readily available, thus making it the most widely studied materials for producing graphene-polymer composite. The most common types of polymer used for synthesis of graphene based polymer composites is an intrinsically conducting polymers (ICPS). ICPs are a new class of polymers having a high electronic conductivity in the partially oxidized state that has been discovered by three collaborating scientists, Alan J. Heeger, Alan G. MacDiarmid, and Hideki Shirakawa. ${ }^{145}$ One of the advantages possess by ICPs is that it is easy to synthesize through chemical or electrochemical process which produces a stable and reproducible dispersion. Moreover, other remarkable advantages of using ICPs is that it provides an excellent signal transduction for molecular detection due to its high sensitivity in their conductivity and electrochemical activity towards molecular interaction, thus opening possibilities for biosensor applications. ${ }^{146}$ A number of ICPs and its derivative have been used to developed graphene based polymer modified electrodes for the detection of DA including polyvinylpyrrolidone (PVP), ${ }^{147}$ poly(3,4-ethylenedioxythio-phene) (PEDOT) ${ }^{148}$ polystyrene (PS), ${ }^{149}$ polyaniline (PANI), ${ }^{150}$ polyethyleneimine $(\mathrm{PEI}),{ }^{119}$ polypyrrole (PPy), ${ }^{151}$ 5,15-pentafluorophenyl-10,20- $p$ - aminophenylporphyrin (porphyrin), ${ }^{\mathbf{1 5 2}}$ chitosan, ${ }^{\mathbf{1 5 3}}$ and Nafion. ${ }^{\mathbf{1 5 4}}$ Manivel $^{137}$ and Liu and co-workers ${ }^{142}$ has modify a GCE using PANI hybridized with GO and graphene for the detection of DA using in situ polymerization technique. The results prove that the PANI/graphene modified electrode has showed excellent catalytic activity towards electrochemical oxidation of DA as compared to the PANI/GO modified electrode. This may be due to the non-conductive property of GO that could not accelerate the electron transfer; hence revealing a weak current response for DA determination.

Various synthetic routes have recently been reported for the fabrication of graphene-polymer based composite including together-blending via ultrasonication, ${ }^{\mathbf{1 0 5 , 1 5 5}}$ heat treatment, ${ }^{\mathbf{1 5 6}}$ microwave assisted chemical reduction, ${ }^{\mathbf{1 1 9}}$ and electropolymerization techniques. ${ }^{\mathbf{1 4 7 , 1 5 7}}$ Electropolymerization method has been paid the most attention because it permits the regulation of the spatial location and selective control of the material properties through variation of the polymer monomer, the potential window, and the electrolytic conditions during the electrodeposition process. ${ }^{\mathbf{1 5 8}}$ In addition, this method also provides a simple, fast, and direct procedure. A report by Liu et al. described the electropolymerization process to fabricate PVP/graphene on top of GCE surface. ${ }^{147}$ A drop of GO solution was dropped on the clean GCE surface before CV scans was carried out for five times. Then, the process continued by drop casting the PVP solution on top of the graphene/GCE surface to form PVP/graphene/GC modified electrode. Likewise, Liu and co-workers $^{\mathbf{1 5 7}}$ developed a poly(o-phenylenediamine) (PoPD)/ graphene hybrid composite using electropolymerization technique where electropolymerization of $o$-phenylenediamine was performed on the graphene/GC electrode. This modified electrode was then used as a working electrode in the electrochemical cell for the detection of DA in human urine samples. The carbon paste electrode (CPE) was also frequently used in sensor application. A novel technique to prepare a graphene/ 
chitosan nanocomposite modified on the surface of CPE has been reported by Chang et al. ${ }^{155}$ The reduction process of graphite was done by adding graphite flakes in a mixture of $N, N$-dimethylformamide and acetic acid via ultrasonication. Next, the chitosan was added in the mixture to form graphene/ chitosan composite, before being used to modify the CPE. Han et al. had synthesized graphene/chitosan composite by a together-blending and in situ chemical reduction method. ${ }^{76}$ This composite was then being used to modify GC electrode for determination of DA. The result shows that the modified CPE exhibited an improved DA sensing compared to the modified GC electrode. Based on these studies, due to the expensive manufacturing cost of GCE and better sensing performance of modified CPE, graphene/polymer composite modified CPE has emerged as one of the potential working electrode to replace graphene/polymer composite modified GCE in the future for DA sensor application.

Yan and co-workers ${ }^{\mathbf{1 5 9}}$ reported a novel high-performance organic electrochemical transistors (OECT) based DA sensor with functionalized gate electrodes. The selectivity of the fabricated biocompatible polymers (Nafion or chitosan) modified gate electrodes was significantly improved with the help of Nafion or chitosan which can be attributed to the different electrostatic interactions between the polymer films and the analytes. Meanwhile, the sensitivity of the devices was improved by using graphene nanomaterials including graphene and rGO because of the excellent charge transport properties and high surface to volume ratio of graphene flakes. The devices modified with Nafion-graphene showed the detection limit down to 5 $\mathrm{nM}$ with broader linear range from $5 \mathrm{nM}$ to $1 \mu \mathrm{M}$ and excellent selectivity towards DA. A summary of the various graphenepolymer composites based electrochemical sensor in the detection of DA can be referred in Table 4 .

T. Qian and co-workers ${ }^{139}$ had used RGO-HDPPy modified GC electrode for DA analysis in human blood serum and the result revealed 99.4 to $102.4 \%$ recovery. Meanwhile, a novel electrochemical aptasensor of GR-PANI modified GC electrode fabricated by S. Liu et al. ${ }^{138}$ shows good recovery values from 99 to $105 \%$ after tested in human blood serum. Other than that, the PVP/GR/GCE was also applied in the determination of DA (urine samples) that displays 97.9 to $101.4 \%$ recovery. ${ }^{135}$ The applicability of these electrode for determination of DA in real samples were fairly good, hence it possesses an excellent potential in sensor application.

Obviously, among the reported works, the GR/PANI modified electrode reveals a significantly low LOD of $1.98 \mathrm{pM}$ for DA

Table 4 Comparison of various graphene-polymer composite modified electrodes towards the detection of dopamine ${ }^{a}$

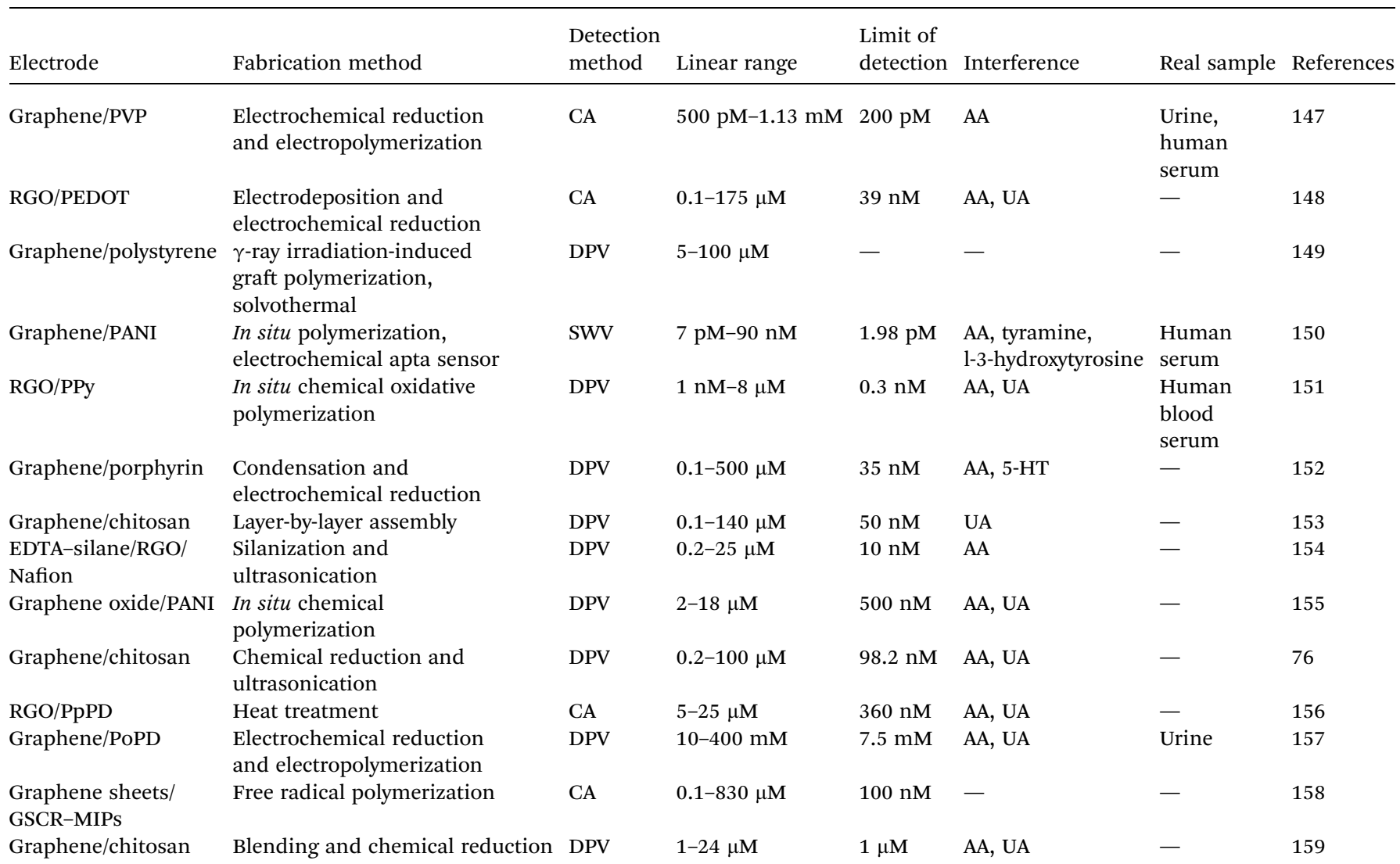

${ }^{a}$ PVP: polyvinylpyrrolidone; PANI: polyaniline; PPy: polypyrrole; PEDOT: poly(3,4-ethylenedioxythiophene); PpPD: poly( $p$-phenylenediamine); PoPD: poly(o-phenylenediamine); GSCR-MIPs: Congo red-molecular imprinted polymers; EDTA-silane: $N$-(trimethoxysilylpropyl) ethylenediamine triacetic acid. 
detection and have shown an excellent potential for the practical application in DA analysis with 99 to $105 \%$ recovery after tested in human blood serum sample. This excellent result was due to the presence of an aptamer in this novel label-free electrochemical aptasensor that is so well attached onto the GR-PANI surface which is used in capturing DA molecules. The interaction between the aptamer and DA molecules will induce a current response which is transferred through an excellent electron transfer activity provided by the GR-PANI nanocomposite. Therefore, this action will increase the sensitivity and selectivity of the sensor which is able to provide a very low LOD in the $\mathrm{pM}$ range.

\subsection{Graphene-carbonaceous composite as electrochemical sensor}

Carbonaceous materials have received considerable interest to be used as electrode materials owing to their low cost, good electron transfer kinetics, and biocompatibility. Since its discovery in 1991 by Ijima et al. ${ }^{\mathbf{1 6 0}}$ carbon nanotubes (CNTs) are viewed as rolled version of graphene sheets which are one of the exciting materials for fabricating electrochemical biosensors. CNTs have shown good electrocatalytic properties in the oxidation and reduction of a wide variety of compounds that can be attributed to their unique properties including enhanced electronic properties, large edge lane/basal plane ratio, outstanding mechanical strength, and rapid electrode kinetics. In order to overcome the shortcomings of poor sensitivity and selectivity properties of modified electrodes, several approaches have been developed into forming graphene-CNT composites to achieve synergistic integration of graphene and CNT. All of these studies found out that the graphene-CNT composites could enhance sensitivity, achieve low detection limit, and provide better electron transfer kinetics.

Nickel foam is a fascinating material that can be used as a growth substrate due to its high biocompatibility. As reported by Dong et al. ${ }^{161}$ the electrochemical DA sensor was fabricated based on a 3D graphene-CNT hybrid foam synthesized using two-step chemical vapour deposition (CVD) approach under atmospheric pressure. Serving as a free-standing electrode, their 3D hybrid electrode results in an excellent amperometric signals for detection of DA with a low detection limit of $20 \mathrm{nM}$ $(S / N=9.2)$ and high sensitivity of $470.7 \mathrm{~mA} \mathrm{M}^{-1} \mathrm{~cm}^{-2}$. In addition, the material was also applied for the determination of $\mathrm{H}_{2} \mathrm{O}_{2}$, which can be indirectly detected with the assistance of enzymes. Interestingly, they have modified the 3D hybrid electrode with horseradish peroxidase (HRP) and Nafion which results in an amperometric response of high sensitivity (137.9 $\left.\mathrm{mA} \mathrm{M}{ }^{-1} \mathrm{~cm}^{-2}\right)$, low detection limit of $1 \mu \mathrm{M}(S / N=17.4)$, and a wide linear detection range $(10 \mu \mathrm{M}-1 \mathrm{mM})$. In the case of electrochemical detection of DA with the co-existence AA and UA, Sun et al. ${ }^{\mathbf{1 6 2}}$ developed core-shell MWCNT-graphene oxide nanoribbons (GONRs)-modified GCE for the simultaneous detection of the DA, AA, and UA. The GONRs was fabricated by the microwave-assisted facile unzipping of MWCNTs producing powder form. To prepare the modified electrode, the powder was mixed with deionized water, ethanol, and Nafion. The well- resolved corresponding peak potential separations of $229.9 \mathrm{mV}$ (AA to DA), $126.7 \mathrm{mV}$ (DA to UA), and $356.6 \mathrm{mV}$ (AA to UA) were achieved. In addition, the amperometric response displayed a low LOD for AA, DA, and UA as 60, 80, and $70 \mathrm{nM}$, respectively.

In another study involving quantum dots, it has been used in many bio-analytical applications due to their large surface area and good stability. Hu et al. ${ }^{\mathbf{1 6 3}}$ fabricated reduced graphene oxide (rGO)/carbon dot (CD) composite film for highly selective and sensitive detection of DA with the interference of AA and UA. CDs are novel due to their zero-dimensional carbon materials which could effectively prevent the aggregation of graphene. The composite was prepared by adding GO solution to $\mathrm{CD}$ solution and placed into an autoclave for $4 \mathrm{~h}$ at $90^{\circ} \mathrm{C}$. In this process, CDs acts as the reducing agent which can reduce GO to rGO and were applied to GCE using a micro injector. By using DPV analysis technique, the composite responded linearly to DA in a wide concentration range from 0.01 to $450.0 \mu \mathrm{M}$ with a low detection limit of $1.5 \mathrm{nM}$. In addition, the composite also showed high stability which could keep its activity for at least two weeks. Interestingly, the addition AA and UA still did not interfere in the DA determination capability. This is due to the cationic exchanger of $\mathrm{rGO} / \mathrm{CDs} / \mathrm{GCE}$ that selectively attracts cationic DA and prevents anionic AA and UA from reaching the GCE surface. Likewise, Chen et al. ${ }^{164}$ reported the simultaneous detection of acetaminophen (APAP) and DA at a single walled carbon nanotube (SWCNT)/graphene nanosheet (GNS) hybrid modified films. The films were prepared by sonicating the suspension of a mixture of SWCNT and GNS in DMF solvent and were subsequently dropped onto the GCE surface. The simultaneous determination of both analytes exhibits selectivity properties in which the hybrid film managed to detect APAP from the interference of AA. The peak currents for both analytes increased linearly in the concentration range from 0.1-52.5 $\mu \mathrm{M}$ for APAP and DA. Hence, the detection limit $(S / N=3)$ was estimated to be $13 \mathrm{nM}$ for APAP and $10 \mathrm{nM}$ for DA.

Besides graphene-CNT composites, GO-CNT composite was also successfully applied for the determination of DA. Although the conductivity of GO, a 2D oxygenated graphene sheet is not as high as graphene, it is regarded as a suitable material for biosensing analysis. GO shows good hydrophilicity properties and could disperse in water due to its abundant hydrophilic functional groups, such as $\mathrm{OH}, \mathrm{COOH}$, and epoxides on the basal plane and the sheet edge, all of which can be orthogonally functionalized for biomolecular detection. Moreover, it can also have a good electronic property which carries electron from insulator to low band gap semiconductor depending upon its degree of oxidation. Cheemalapati et al. ${ }^{\mathbf{1 6 5}}$ prepared $\mathrm{GO} /$ MWCNT nanocomposite by sonicating the mixture of MWCNT and GO into water to produce a homogeneous dispersion. In this work, GO/MWCNT composite was applied for the simultaneous determination of DA and paracetamol (PA). The measurements were made through CV, DPV, and CA as shown in Fig. 14 in which the modified electrode exhibits higher electrocatalytic oxidation of DA and PA in the linear response range from 0.2 to $400 \mu \mathrm{M}$ and 0.5 to $400 \mu \mathrm{M}$ with a low detection limit of 22 and $47 \mathrm{nM}$, respectively. 

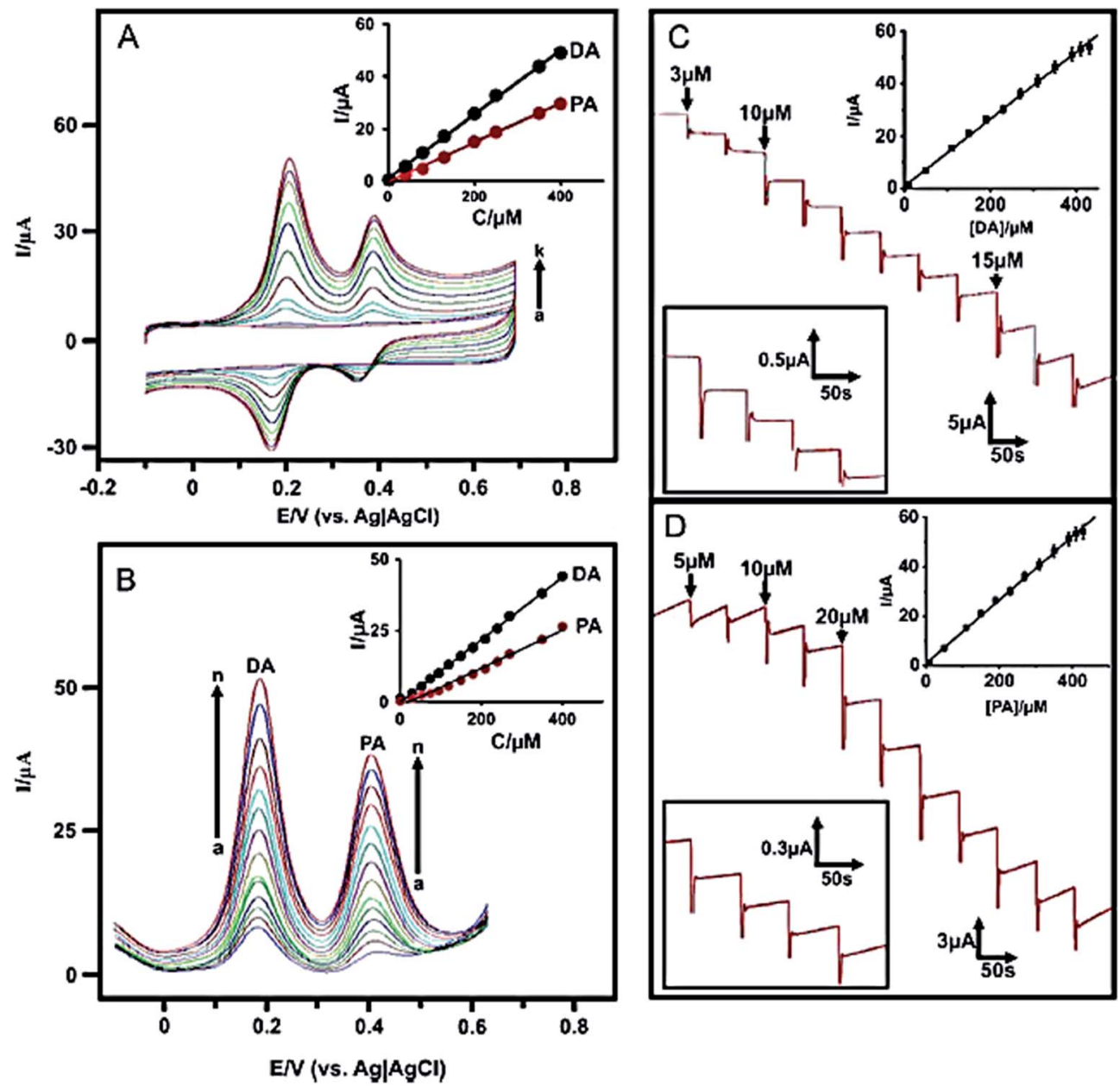

Fig. 14 (A) CVs of MWCNT/GO nanocomposite film modified GCE (a) without and (b-k) with 200 nM-400 mM of DA and PA containing deoxygenated PBS at $50 \mathrm{mV} \mathrm{s}^{-1}$ scan rate. Inset plot shows the linear dependence of Ipa vs. [DA] and [PA]. (B) DPVs recorded at MWCNT/GO nanocomposite film modified GCE (a) without and (b-n) with $200 \mathrm{nM}-400 \mathrm{mM}$ of DA and PA containing deoxygenated PBS at $50 \mathrm{mV} \mathrm{s}^{-1}$ scan rate. Inset plot shows the linear dependence of Ipa vs. [DA] and [PA]. (C) Amperometric $i-t$ response at MWCNT/GO nanocomposite film modified rotating disc GCE upon successive additions of $100 \mathrm{nmol} \mathrm{L}-1-400 \mathrm{mM}$ of DA into continuously stirred N2 saturated PBS (pH $=7$ ). Applied potential: $0.204 \mathrm{~V}$; inset (upper) shows the calibration curve of Ipa vs. [DA]. Inset (bottom) shows $i-t$ response for each addition of 1 mM of DA. (D) Amperometric responses acquired under similar conditions for successive additions of $200 \mathrm{nM}-400 \mathrm{mM}$ of PA. Applied potential of $0.408 \mathrm{~V}$; inset (upper) shows the calibration curve of Ipa vs. [PA]. Inset (bottom) shows $i-t$ response for each addition of $1 \mathrm{mM}$ of PA. Reprinted with permission from ref. 154, Copyrights (2013) Elsevier Publications.

Another work that simultaneously detect DA and other interference species is done by Yang et al. ${ }^{166}$ They have developed hexadecyltrimethylammonium bromide (CTAB) functionalized GO/MWCNT modified GCE (CTAB-GO/MWCNT/ GCE) as a novel system to construct sensing interface to achieve simultaneous detection of DA, AA, UA, and nitrite $\left(\mathrm{NO}_{2}{ }^{-}\right)$. For preparation, they have stirred the mixture of graphene and CTAB and then mixed with MWCNT under ultrasonic agitation. In their DPV studies, the linear calibration plots for AA, DA, UA, and $\mathrm{NO}_{2}{ }^{-}$were obtained over the range of 5-300 $\mu \mathrm{M}, 5-500 \mu \mathrm{M}$, 3-60 $\mu \mathrm{M}$, and 5-800 $\mu \mathrm{M}$ with LOD of $1,1.5,1$, and $1.5 \mu \mathrm{M}$, respectively. Zhang et $a l^{78}$ developed a novel biosensor for simultaneous determination of DA, AA, and nitrite $\left(\mathrm{NO}_{2}{ }^{-}\right)$ based on graphene/poly-cyclodextrin/multiwall carbon nanotubes (CDP-GS-MWCNTs) modified GCE. They have fabricated the biosensor by dispersing graphene sheets (GS) and multiwall carbon nanotubes (MWCNTs) in the mixed solution of cyclodextrin (CD) and cyclodextrin prepolymer (pre-CD), which was subsequently dropped on the surface of the GCE. Based on the CV and DPV techniques, a good separation of the oxidation peak potentials for $\mathrm{AA}, \mathrm{DA}$ and $\mathrm{NO}_{2}{ }^{-}$was shown and the peak currents increased linearly as the concentrations of $\mathrm{AA}, \mathrm{DA}$ and $\mathrm{NO}_{2}{ }^{-}$were increased. The linear response range for $\mathrm{AA}, \mathrm{DA}$, and $\mathrm{NO}_{2}{ }^{-}$were determined in the ranges of $5 \mu \mathrm{M}-0.48 \mathrm{mM}, 0.15-21.65 \mu \mathrm{M}$, and $5 \mu \mathrm{M}-6.75 \mathrm{mM}$, respectively, with the detection limit of $1.65 \mu \mathrm{M}$, $0.05 \mu \mathrm{M}$, and $1.65 \mu \mathrm{M}$. A summary of the various graphenecarbonaceous materials based electrochemical sensor in the detection of DA can be referred in Table 5 .

The applicability in real sample analysis with the graphenecarbonaceous materials was studied by standard addition method. The GO/MWCNT, CTAB/GO/MWCNT and graphene/ poly-CD/MWCNT have been employed for the detection of DA 
Table 5 Comparison of various graphene-carbonaceous materials modified electrodes towards the detection of dopamine ${ }^{a}$

\begin{tabular}{|c|c|c|c|c|c|c|c|}
\hline Electrode & $\begin{array}{l}\text { Fabrication } \\
\text { method }\end{array}$ & $\begin{array}{l}\text { Detection } \\
\text { method }\end{array}$ & Linear range & $\begin{array}{l}\text { Limit of } \\
\text { detection }\end{array}$ & Interference & Real sample & References \\
\hline 3D graphene/CNT/Nf/HRP & Two-step CVD & $\mathrm{CA}$ & $2-64 \mu \mathrm{M}$ & $20 \mathrm{nM}$ & - & - & 161 \\
\hline $\mathrm{RGO} /$ carbon dots & Reduction & DPV & $\begin{array}{l}10 \mathrm{nM}-450 \\
\mu \mathrm{M}\end{array}$ & $1.5 \mathrm{nM}$ & DA, AA, UA & - & 163 \\
\hline CTAB/GO/MWCNT & Sonication & DPV & $5-500 \mu \mathrm{M}$ & $1.5 \mu \mathrm{M}$ & $\mathrm{AA}, \mathrm{UA}$ & Urine & 166 \\
\hline Graphene/poly-CD/MWCNT & Sonication & DPV & $0.15-22 \mu \mathrm{M}$ & $50 \mathrm{nM}$ & $\begin{array}{l}\mathrm{DA}, \mathrm{AA}, \\
\mathrm{NO}_{2}{ }^{-}\end{array}$ & Human urine & 78 \\
\hline
\end{tabular}

${ }^{a}$ CNT: carbon nanotube; Nf: Nafion; HRP: horseradish peroxidase; SWCNT: single walled carbon nanotube; MWCNT: multiwalled carbon nanotube; CTAB: hexadecyl ammonium bromide; poly-CD: poly-cyclodextrin.

in urine. The recovery of DA in urine using GO/MWCNT, ${ }^{165}$ $\mathrm{CTAB} / \mathrm{GO} / \mathrm{MWCNT},{ }^{166}$ and graphene/poly-CD/MWCNT ${ }^{78}$ were obtained between 98.8 and 104.0, 99 and 110, and 95.0 and $106.6 \%$, respectively. The DA was also determined in human serum sample using SWCNT/graphene NS electrode ${ }^{164}$ with the recovery falling between 97.1 and $102.1 \%$. Thus, the excellent recoveries of DA was achieved more than $94 \%$ validates that this nanocomposite materials remain promising for real sample analysis. Among the reviewed graphene-carbonaceous composite as an electrochemical sensor, the RGO/carbon dots modified electrode showed the lowest LOD of $1.5 \mathrm{nM}$ for DA detection. To date, due to its unique size and shape-dependent of zero-dimensional of CDs which effectively prevents the aggregation of graphene, it should be considered as another significant and important electrochemically active material that may have the potential to be used in sensing applications.

\subsection{Graphene-clay composite as electrochemical sensor}

The typical properties of clay minerals such as the ability of cation exchange, the possibility to incorporate inorganic or organic substances between the interlayers, and the affinity of charged interlayers/interlayers cations for polar the substances, might lead to characteristic changes in the electrochemical properties of the clays, which might be useful for the development of sensors. In the past few years, graphene-clay composites have showed promising results to be used as an electrochemical sensor towards a wide range of biomolecules.

Li et $a .^{77}$ attempted to study the electrochemical performance of hydrotalcite-like multifunctional materials or anionic clays, named as double hydroxides (LDHs)-based composites and used them for fabricating the electrochemical DA sensor. They have explored a facile two-step procedure to prepare NiAlLDH/graphene (NiAl-LDH/G) nanocomposite using a
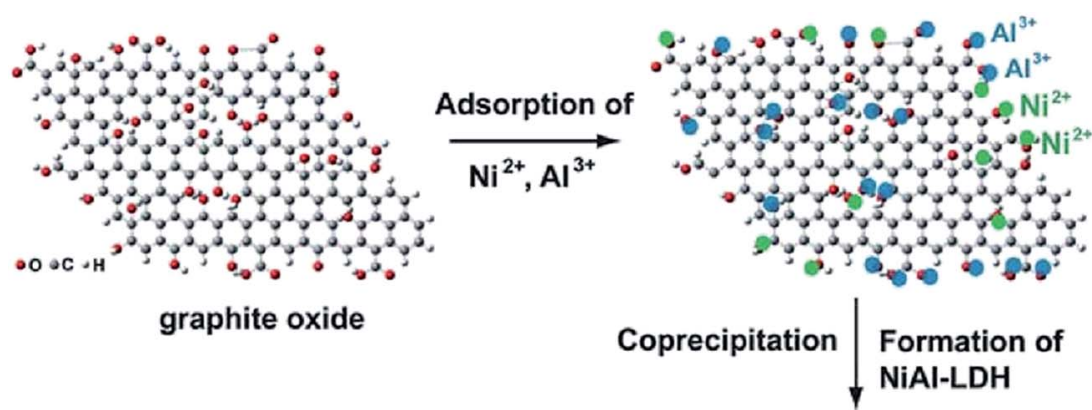

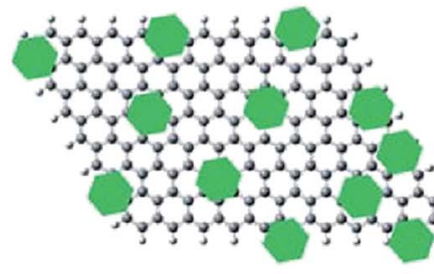

NiAl-LDH/graphene

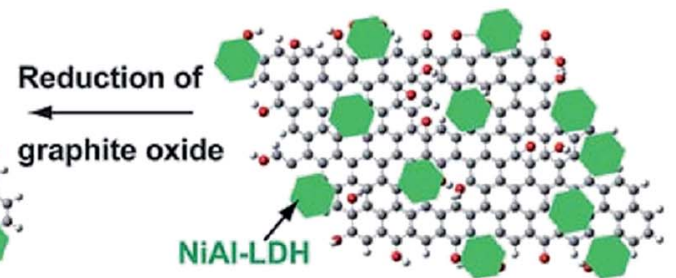

NiAl-LDH/graphite oxide

Fig. 15 Schematic illustration of preparing the nanostructured NiAl-LDH/G composite Reprinted with permission from ref. 157, Copyrights (2011) Royal Society of Chemistry. 
conventional co-precipitation process under low-temperature conditions and subsequent reduction of the supporting GO (Fig. 15).

The NiAl-LDH/G nanocomposite exhibits highly enhanced electrocatalytic activity in comparison with the electrode modified by the pristine NiAl-LDH. Good linear relationships of electrocatalytic redox peak currents responded to DA were obtained in the DA concentration range from 0.5 to $120 \mu \mathrm{M}$ (reduction peak current), and also in the concentration range from 80 to $400 \mu \mathrm{M}$ (oxidation peak current). The detection limits were estimated to be 0.2 and $9.6 \mu \mathrm{M}(S / N=3)$ for the reduction and oxidation peak currents, respectively. Another work also reported by Ding et al. ${ }^{167}$ in which they investigated the possibility to co-immobilized graphene with synthetic lamellar clay, Laponite. They have developed a Laponite/graphene (Lap/G)modified GCE for the simultaneous detection of DA, AA, and UA (Fig. 16). The composite was prepared by directly exfoliating graphite with Laponite under mechanical grinding. The wellseparated voltammetric peaks of DA, AA, and UA were observed at the Lap/G/GCE as compared with bare GCE in CV as well as in DPV techniques. Through their DPV techniques, the linear response for determination of AA, DA, and UA, ranges from 5 to $1300 \mu \mathrm{M}, 0.5$ to $170 \mu \mathrm{M}$, and 0.1 to $26 \mu \mathrm{M}$, respectively. Therefore, the respective detection limits were determined at $2.2 \mu \mathrm{M}, 0.25 \mu \mathrm{M}$, and $45 \mathrm{nM}$.

As compared to the NiAl-LDH/G, Lap/G reveals a lower LOD in detecting DA. In addition, the reproducibility and stability of both materials are also examined. Lap/G showed better reproducibility of electrode in which the Lap/G could detect around $10 \mu \mathrm{M}$ DA using five independently prepared electrodes with a RSD of $3.0 \%$, while NiAl-LDH/G detected around $100 \mu \mathrm{M}$ DA using five independently prepared electrodes with the interassay coefficient of variation of $4.2 \%$.
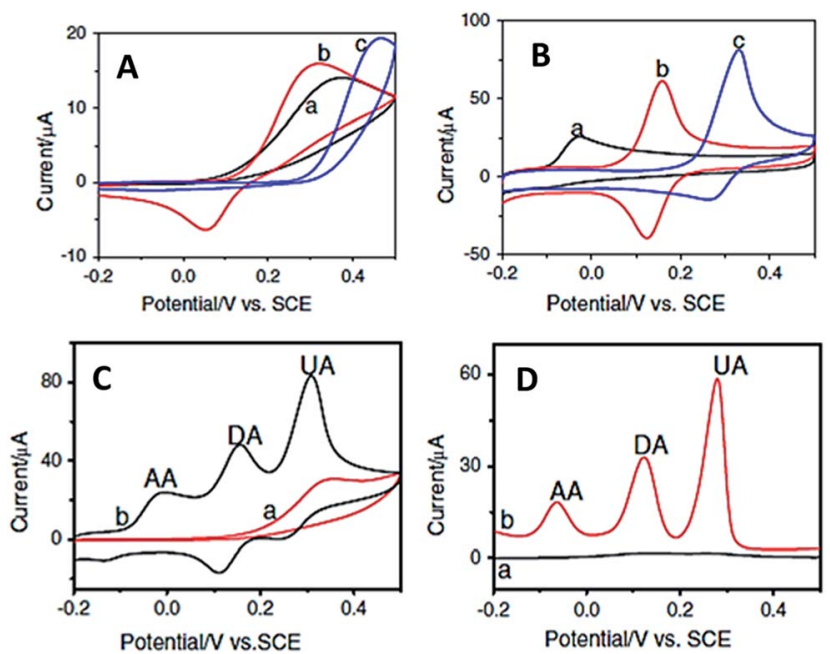

Fig. $16 C V$ s obtained for $1 \mathrm{mM}$ of $A A(a), D A(b)$, and $U A(c)$ individually in $0.1 \mathrm{M} \mathrm{PBS}$ at (A) bare GCE and (B) Lap/G/GCE. (C) CVs and (D) DPVs obtained for mixture of $1 \mathrm{mM} \mathrm{AA}, 0.7 \mathrm{mM} \mathrm{DA}$, and $1 \mathrm{mM} \cup \mathrm{A}$ in $0.1 \mathrm{M}$ PBS ( $\mathrm{pH} \mathrm{7.0)}$ ) at bare GCE (a) and Lap/G/GCE (b). Reprinted with permission from ref. 158, Copyrights (2014) Springer.

\subsection{Graphene-zeolite composite as electrochemical sensor}

Zeolites are crystalline aluminosilicates with pores and channels of molecular dimensions. The unique properties of microporous zeolites crystalline, including ion-exchange properties, adsorption, molecular sieving, catalysis, and conductivity have been exploited in improving the electrocatalytic activity of biosensors. Therefore, the fabrication of composite materials of zeolite with graphene yields a more promising prospect for the electrochemical detection of biologically important molecules. He et al. ${ }^{168}$ reported a one pot synthetic method for the preparation of copper $(\mathrm{Cu})$ nanoparticles doped-zeolite $\mathrm{A} /$ graphene (CuZEA/rGO) via reduction of $\mathrm{Cu}^{2+}$ functionalized zeolite A and GO in. The simultaneous determination of both DA and AA measured via a DPV technique through the oxidation peaks at the CuZEA/rGO/GCE was relatively concentration independent. The electrocatalytic oxidation currents of DA and AA were linearly related to the corresponding concentration in the range of 0.1 to $19 \mu \mathrm{M}$ for DA and 20 to $200 \mu \mathrm{M}$ for AA. The detection limit $(S / N=3)$ were estimated to be at $41 \mathrm{nM}$ for DA and $11 \mu \mathrm{M}$ for AA. Likewise, Wang et al. ${ }^{169}$ reported the simultaneous DA and AA biosensor based on $\mathrm{Cu}$ nanoparticles doped zeolitegraphene (CuZEO/rGO) modified electrode, prepared by mixing copper(II) functionalized zeolite A with GO as the precursor and subsequent co-reduction by reducing chemical agents. The calibration curves for DA and AA were obtained in the range of 0.1 to $19 \mu \mathrm{M}$ and 20 to $190 \mu \mathrm{M}$, respectively. The lowest detection limits $(S / N=3)$ were evaluated as $41 \mathrm{nM}$ and $11 \mu \mathrm{M}$ for DA and AA, respectively. Based on the work presented, the potential difference between the oxidation peaks of AA and DA was adequate in distinguishing AA from DA. Both CuZEA/rGO/GCE and CuZEO/rGO/GCE offers a good possibility for the analysis of AA and DA in clinical use and trials. However, further investigation such as the reproducibility and stability of the modified electrodes still need to be provided in order to verify this potential material.

\subsection{Graphene-metal-organic frameworks as electrochemical sensor}

The novel class of porous materials, metal-organic frameworks (MOFs) is often compared to zeolites for their large internal surface area, extensive porosity, and high degree of crystallinity. MOFs were shown to possess superior adsorption properties as compared to classical adsorption materials. Recently, the hybridization of MOFs and graphene have attracted great interest due to the unique advantages that was offered in this material. Yang et al. ${ }^{81}$ reported the synthesis of graphene supported chromium carbide $(\mathrm{Cr}-\mathrm{G})$ material for electrochemical oxidation and DA detection. The composite made from chromium carbide $\left(\mathrm{Cr}_{3} \mathrm{C}_{2}\right)$ and chemically converted graphene (G) was synthesized by calcination of a Cr-based metal-organic framework (MIL-101) embedded with GO. The MIL-101 embedded with graphene oxide (MGO) was synthesized by the self-assembly process of $\mathrm{Cr}\left(\mathrm{NO}_{3}\right)_{3} \cdot 9 \mathrm{H}_{2} \mathrm{O}$ and terephthalic acid in the presence of exfoliated GO through a hydrothermal route. The Cr-G modified electrode (Cr-G/GCE) showed excellent response to the electro-oxidation of DA. The Cr-G/GCE 
maintained the linear growth of the anodic current with the square root of the scan rate from 20 to $300 \mathrm{mV} \mathrm{s}^{-1}$, indicating that the electrochemical behavior of $\mathrm{Cr}-\mathrm{G} / \mathrm{GCE}$ for DA oxidation is a diffusion-controlled process. SWV technique shows the current increase linearly with the increase of the DA concentration in the range 1-10 and 10-100 $\mu \mathrm{M}$, implying the potential use of $\mathrm{Cr}-\mathrm{G} / \mathrm{GCE}$ as a DA biosensor. Another work published recently by Wang et al. ${ }^{\mathbf{1 7 0}}$ demonstrates the facile ultrasonication method for the preparation of a novel hybrid nanocomposite of copper terephthalate MOF-graphene oxide

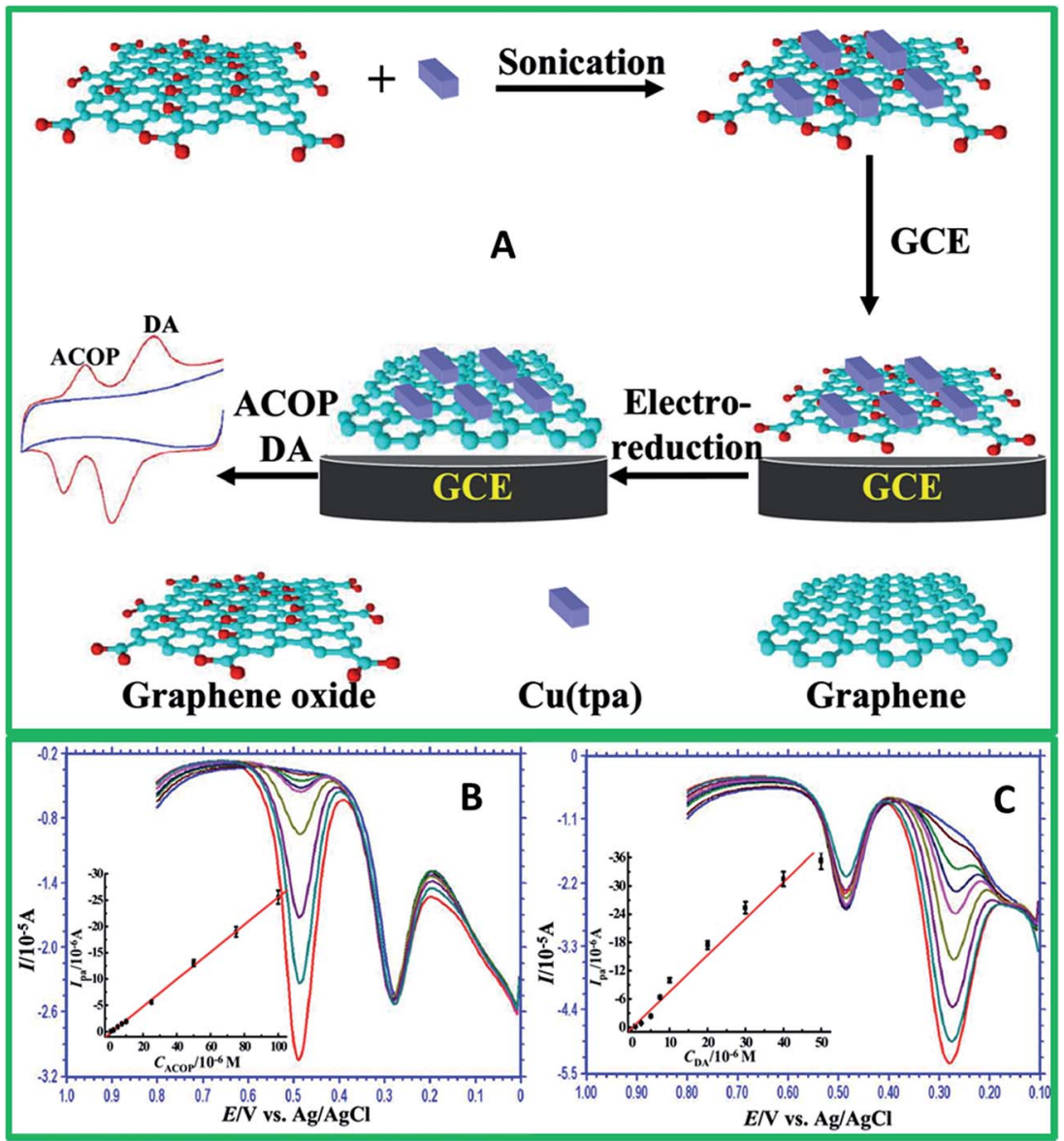

Fig. 17 (A) Schematic illustration for the sonication-assisted preparation of $\mathrm{Cu}(\mathrm{tpa})-\mathrm{GO}$, and its application for the simultaneous determination of ACOP and DA. (B) DPVs of ACOP over a concentration range of 1.0 to $100 \mu \mathrm{M}$ at Cu(tpa)-EGR/GCE in the presence of $20 \mu \mathrm{M}$ DA in $0.1 \mathrm{M}$ PBS ( $\mathrm{pH}$ 5.0). (C) DPVs of DA over a concentration range of 1.0 to $50 \mu \mathrm{M}$ at $\mathrm{Cu}(\mathrm{tpa})-\mathrm{EGR} / \mathrm{GCE}$ in the presence of $50 \mu \mathrm{M} \mathrm{ACOP}$ in $0.1 \mathrm{M}$ PBS (pH 5.0). Insets in (B) and (C) are respectively the linear calibration plots of peak currents (Ipa) versus the concentration of ACOP (CACOP) and DA (CDA). Reprinted with permission from ref. 162, Copyrights (2014) American Chemical Society.

Table 6 Comparison of some rare graphene based composites modified electrodes towards the detection of dopamine ${ }^{a}$

\begin{tabular}{|c|c|c|c|c|c|c|c|}
\hline $\begin{array}{l}\text { NiAl-layered double hydroxide/ } \\
\text { graphene }\end{array}$ & Co-precipitation & $\mathrm{CV}$ & $80-400 \mu \mathrm{M}$ & $9.6 \mu \mathrm{M}$ & DA, AA & - & 77 \\
\hline Laponite/graphene & Mechanical grinding & $\mathrm{CV}$ & $0.5-170 \mu \mathrm{M}$ & $0.25 \mu \mathrm{M}$ & DA, AA, UA & - & 167 \\
\hline $\mathrm{Cu}$ NPs doped-zeolite A/graphene & $\begin{array}{l}\text { Magnetic stirring and chemical } \\
\text { reduction }\end{array}$ & $\mathrm{CV}$ & $0.1-19 \mu \mathrm{M}$ & $41 \mathrm{nM}$ & $\mathrm{DA}, \mathrm{AA}$ & - & 168 \\
\hline Copper terephthalate MOF-GO & Ultrasonication & DPV & $1-5 \mu \mathrm{M}$ & $0.21 \mu \mathrm{M}$ & DA, ACOP & $\begin{array}{l}\text { Urine, } \\
\text { serum }\end{array}$ & 170 \\
\hline
\end{tabular}

${ }^{a} \mathrm{Cu}$ NPs: copper nanoparticles; MOF: metal organic frameworks. 
$(\mathrm{Cu}(\mathrm{tpa})-\mathrm{GO})$ for the simultaneous determination of acetaminophen (ACOP) and DA. The electrochemical sensing behavior was investigated by electrochemical reduction of $\mathrm{Cu}$ (tpa)-GO coated GCE, since GO is converted to high conductive electrochemically reduced graphene (EGR) (Fig. 17(A)). The Cu(tpa)-EGR/GCE showed significantly improved electrochemical signals and peak profiles for the both analytes as compared to $\mathrm{Cu}(\mathrm{tpa})-\mathrm{GO} / \mathrm{GCE}$, owing to the synergistic effect from high conductivity of EGR and unique electron mediating action of $\mathrm{Cu}(\mathrm{tpa})$. DPV exhibited the oxidation peak currents of ACOP and DA that were linearly correlated to their concentration in the ranges of 1-100 $\mu \mathrm{M}$ and 1-50 $\mu \mathrm{M}$, respectively (Fig. 17(B) and (C)). The detection limits for ACOP and DA were estimated to be as low as 0.36 and $0.21 \mu \mathrm{M}$, respectively.

For real sample analysis, $\mathrm{Cu}(\mathrm{tpa})-\mathrm{EGR} / \mathrm{GCE}^{\mathbf{1 7 0}}$ was used to determine DA in diluted urine and serum samples for the recovery of the spiked samples ranged between 98 and 101\%, indicating that the material was reliable and sensitive enough for the determination of ACOP and DA in real samples. A summary of the some rare graphene composites with clay, zeolite, and MOF based electrochemical sensor for the detection of DA can be referred in Table 6. Among them, the Cu NPs doped-zeolite A/graphene modified electrode showed the lowest LOD of $45 \mathrm{nM}$ for DA.

\section{Conclusions and future outlook}

This review article has described the recent publications in the development of electrochemical sensing of DA using graphene and their nanocomposites based materials. Some possible future directions for the electrochemical sensing of DA using electroanalytical techniques are for the wider implementation of graphene-based materials for the tuning of DA sensing. Although the unique properties of graphene-based materials have offered many advantages for the electrochemical sensing of DA, further efforts should be made focusing on: (i) understanding the interaction between graphene and DA, (ii) improved sensitivity and selectivity of the modified electrodes in the detection of DA, (iii) long term stability and reproducibility of the modified electrodes, and (iv) the extending of application to practicality of the modified electrodes to real sample analysis in human urine, blood serum, and pharmaceutical injections.

The main challenge faced related with graphene-based materials in electrochemical sensors, is the selectivity towards DA in the presence of other important biological analytes and significant discoveries in this field are expected, due to the increasing need for portable sensors in a wide range of biological and clinical fields. Despite these challenges, the excellent electronic conductivity of graphene-based materials and their ability to combine with other materials makes them ideal candidates for making electrochemical sensor devices. Major fundamental and technological advances have been utilized to enhance the capabilities and improve the detection limit and specific detection of DA in devices fabrication which is easy to operate, reusable, and low-cost fabrication.
Many new graphene-based materials have been investigated, for example graphene, $\mathrm{N}$-doped graphene, functionalized graphene, graphene-metal nanoparticles, graphene-metal oxides, graphenepolymer composites, graphene-carbonaceous composite, graphene-clay composite, graphene-zeolite composite, graphenemetal organic framework have been used for sensor construction to improve their performance of DA sensing. However, only the graphene combined with polymer have shown the capability as a promising material for the electrochemical sensing of DA due to the simple preparation, high sensitivity and selectivity, good reproducibility, and long-term stability. In conclusion, the graphene-based materials and its unique electronic properties make excellent sensing material in the fabrication of DA sensor. In the near future, new developments in the graphene-based composite materials are required in the field of electrochemical sensing of DA with high sensitivity and selectivity for making sensor devices for practical applications. The graphene-based materials and its modified electrode is a versatile system for the detection of DA in the near future.

\section{Acknowledgements}

This work was financially supported by a University of Malaya Research Grant Programme (RP007C/13AFR) and the High Impact Research Grant from the Ministry of Higher Education of Malaysia (UM.C/625/1/HIR/MOHE/SC/21).

\section{References}

1 K. Jackowska and P. Krysinski, Anal. Bioanal. Chem., 2013, 405, 3753-3771.

2 T. Mele, M. Čarman-Kržan and D. M. Jurič, Int. J. Dev. Neurosci., 2010, 28, 13-19.

3 H. Lövheim, Med. Hypotheses, 2012, 78, 341-348.

4 E. Ling, R. A. Shrikhande and G. M. Pasinetti, in Encyclopedia of Neuroscience, ed. L. R. Squire, Academic Press, Oxford, 2009, pp. 1073-1083.

5 M. A. Kurian, P. Gissen, M. Smith, S. J. Heales and P. T. Clayton, Lancet Neurol., 2011, 10, 721-733.

6 S. G. Amara and M. J. Kuhar, Annu. Rev. Neurosci., 1993, 16, 73-93.

7 S. S. Castro, R. J. Mortimer, M. F. De Oliveira and N. R. Stradiotto, Sensors, 2008, 8, 1950-1959.

8 M. Mazloum-Ardakani, H. Rajabi, H. Beitollahi, B. Mirjalili, A. Akbari and N. Taghavinia, Int. J. Electrochem. Sci., 2010, 5, 147-157.

9 J.-W. Mo and B. Ogorevc, Anal. Chem., 2001, 73, 1196-1202. 10 A. Galvan and T. Wichmann, Clin. Neurophysiol., 2008, 119, 1459-1474.

11 M. Mohammadi and S. Akhondzadeh, Acta Med. Iran., 2011, 49, 487-498.

12 T. Obata, J. Neural Transm., 2002, 109, 1159-1180.

13 G. Di Chiara, V. Bassareo, S. Fenu, M. A. De Luca, L. Spina, C. Cadoni, E. Acquas, E. Carboni, V. Valentini and D. Lecca, Neuropharmacology, 2004, 47, 227-241.

14 X. Xu, H. Zhang, H. Shi, C. Ma, B. Cong and W. Kang, Anal. Chem., 2012, 427, 10-17. 
15 V. Carrera, E. Sabater, E. Vilanova and M. A. Sogorb, J. Chromatogr. B: Anal. Technol. Biomed. Life Sci., 2007, 847, 88-94.

16 K. Vuorensola, H. Sirén and U. Karjalainen, J. Chromatogr. B: Anal. Technol. Biomed. Life Sci., 2003, 788, 277-289.

17 Y. Liu, J. Huang, H. Hou and T. You, Electrochem. Commun., 2008, 10, 1431-1434.

18 N. Boes and H. Züchner, J. Less-Common Met., 1976, 49, 223-240.

19 E. Paleček, Talanta, 2002, 56, 809-819.

20 Y. Shao, J. Wang, H. Wu, J. Liu, I. A. Aksay and Y. Lin, Electroanalysis, 2010, 22, 1027-1036.

21 S. Joo and R. B. Brown, Chem. Rev., 2008, 108, 638-651.

22 Y. Wang, H. Xu, J. Zhang and G. Li, Sensors, 2008, 8, 20432081.

23 D. Huang, C. Chen, Y. Wu, H. Zhang, L. Sheng, H.-J. Xu and Z.-D. Liu, Int. J. Electrochem. Sci., 2012, 7, 5510-5520.

24 W. Cai, T. Lai, H. Du and J. Ye, Sens. Actuators, B, 2014, 193, 492-500.

25 A. Hubbard, J. Stickney, M. Soriaga, V. Chia, S. Rosasco, B. Schardt, T. Solomun, D. Song, J. White and A. Wieckohski, J. Electroanal. Chem. Interfacial Electrochem., 1984, 168, 43-66.

26 L. Yang, D. Liu, J. Huang and T. You, Sens. Actuators, B, 2014, 193, 166-172.

27 A. A. Ensafi, M. Taei and T. Khayamian, J. Electroanal. Chem., 2009, 633, 212-220.

28 M.-S. Hsu, Y.-L. Chen, C.-Y. Lee and H.-T. Chiu, ACS Appl. Mater. Interfaces, 2012, 4, 5570-5575.

29 G. A. Evtugyn, R. V. Shamagsumova, R. R. Sitdikov, I. I. Stoikov, I. S. Antipin, M. V. Ageeva and T. Hianik, Electroanalysis, 2011, 23, 2281-2289.

30 T. Selvaraju and R. Ramaraj, J. Electroanal. Chem., 2005, 585, 290-300.

31 N. F. Atta, M. F. El-Kady and A. Galal, Sens. Actuators, B, 2009, 141, 566-574.

32 R. N. Goyal, D. Kaur and A. K. Pandey, Open Chem. Biomed. Methods J., 2010, 3, 115-122.

33 K. Zangeneh Kamali, A. Pandikumar, N. M. Huang, B. H. Ong and H. N. Lim, Sci. World J., 2014, 2014, 396135.

34 B. Fang, G. Wang, W. Zhang, M. Li and X. Kan, Electroanalysis, 2005, 17, 744-748.

35 B. H. Gao, S. N. Ding, Q. Q. Li, D. Shan, Y. M. Sun and S. Cosnier, Electroanalysis, 2012, 24, 1267-1271.

36 H. Qiu, M. Zheng, S. Weng, Z. Huang, R. Xian and X. Lin, Anal. Methods, 2014, 6, 7923-7927.

37 K. Fooladsaz, M. Negahdary, G. Rahimi, A. HabibiTamijani, S. Parsania, H. Akbari-dastjerdi, A. Sayad, A. Jamaleddini, F. Salahi and A. Asadi, Int. J. Electrochem. Sci., 2012, 7, 9892-9908.

38 M. V. Deshpande and E. A. H. Hall, Biosens. Bioelectron., 1990, 5, 431-448.

39 A. S. Kumar, P. Swetha and K. C. Pillai, Anal. Methods, 2010, 2, 1962-1968.

40 Q. Huang, S. Hu, H. Zhang, J. Chen, Y. He, F. Li, W. Weng, J. Ni, X. Bao and Y. Lin, Analyst, 2013, 138, 5417-5423.
41 D. Wu, H. Li, X. Xue, H. Fan, Q. Xin and Q. Wei, Anal. Methods, 2013, 5, 1469-1473.

42 E. R. Sabzi, K. Rezapour and N. Samadi, J. Serb. Chem. Soc., 2010, 75, 537-549.

43 B. Habibi, M. Jahanbakhshi and M. H. Pournaghi-Azar, Electrochim. Acta, 2011, 56, 2888-2894.

44 M. C. Henstridge, E. J. F. Dickinson, M. Aslanoglu, C. Batchelor-McAuley and R. G. Compton, Sens. Actuators, $B, 2010,145,417-427$.

45 H. Cheng, H. Qiu, Z. Zhu, M. Li and Z. Shi, Electrochim. Acta, 2012, 63, 83-88.

46 A. Walcarius, T. Barbaise and J. Bessiere, Anal. Chim. Acta, 1997, 340, 61-76.

47 J.-M. Zen and P.-J. Chen, Anal. Chem., 1997, 69, 5087-5093. 48 Y. Li, C. Huangfu, H. Du, W. Liu, Y. Li and J. Ye, J. Electroanal. Chem., 2013, 709, 65-69.

49 S.-M. Chen and W.-Y. Chzo, J. Electroanal. Chem., 2006, 587, 226-234.

50 P.-Y. Chen, R. Vittal, P.-C. Nien and K.-C. Ho, Biosens. Bioelectron., 2009, 24, 3504-3509.

51 R. D. O'Neill, R. A. Grünewald, M. Fillenz and W. J. Albery, Neuroscience, 1982, 7, 1945-1954.

52 P. Raghu, T. M. Reddy, P. Gopal, K. Reddaiah and N. Y. Sreedhar, Enzyme Microb. Technol., 2014, 57, 8-15.

53 C. R. Raj, K. Tokuda and T. Ohsaka, Bioelectrochemistry, 2001, 53, 183-191.

54 E. Popa, H. Notsu, T. Miwa, D. Tryk and A. Fujishima, Electrochem. Solid-State Lett., 1999, 2, 49-51.

55 J. Kang, X.-B. Yin, X. Yang and E. Wang, Electrophoresis, 2005, 26, 1732-1736.

56 N. Plesu, A. Kellenberger, I. Taranu, B. O. Taranu and I. Popa, React. Funct. Polym., 2013, 73, 772-778.

57 H. Tang, P. Lin, H. L. W. Chan and F. Yan, Biosens. Bioelectron., 2011, 26, 4559-4563.

58 W. Choi, I. Lahiri, R. Seelaboyina and Y. S. Kang, Crit. Rev. Solid State Mater. Sci., 2010, 35, 52-71.

59 D. K. Kampouris and C. E. Banks, Chem. Commun., 2010, 46, 8986-8988.

60 S. Wu, Q. He, C. Tan, Y. Wang and H. Zhang, Small, 2013, 9, 1160-1172.

61 A. Ambrosi, C. K. Chua, A. Bonanni and M. Pumera, Chem. Rev., 2014, 14, 7150-7188.

62 W. Yuan, Y. Zhou, Y. Li, C. Li, H. Peng, J. Zhang, Z. Liu, L. Dai and G. Shi, Sci. Rep., 2013, 3, 2248.

63 D. A. Brownson, L. J. Munro, D. K. Kampouris and C. E. Banks, RSC Adv., 2011, 1, 978-988.

64 D. A. Brownson, C. W. Foster and C. E. Banks, Analyst, 2012, 137, 1815-1823.

65 M. Pumera, Chem. Soc. Rev., 2010, 39, 4146-4157.

66 X.-m. Chen, G.-h. Wu, Y.-q. Jiang, Y.-r. Wang and X. Chen, Analyst, 2011, 136, 4631-4640.

67 M. Zhou, Y. Zhai and S. Dong, Anal. Chem., 2009, 81, 56035613.

68 T. Kuila, S. Bose, P. Khanra, A. K. Mishra, N. H. Kim and J. H. Lee, Biosens. Bioelectron., 2011, 26, 4637-4648.

69 C. Shan, H. Yang, J. Song, D. Han, A. Ivaska and L. Niu, Anal. Chem., 2009, 81, 2378-2382. 
70 M. Yang and S. Gong, Chem. Commun., 2010, 46, 57965798.

71 H. Yin, Y. Zhou, Q. Ma, S. Ai, P. Ju, L. Zhu and L. Lu, Process Biochem., 2010, 45, 1707-1712.

72 W. Li, X. Geng, Y. Guo, J. Rong, Y. Gong, L. Wu, X. Zhang, P. Li, J. Xu and G. Cheng, ACS Nano, 2011, 5, 6955-6961.

73 Y. Wang, Y. Li, L. Tang, J. Lu and J. Li, Electrochem. Commun., 2009, 11, 889-892.

74 F. Li, J. Chai, H. Yang, D. Han and L. Niu, Talanta, 2010, 81, 1063-1068.

75 Y. Fan, H.-T. Lu, J.-H. Liu, C.-P. Yang, Q.-S. Jing, Y.-X. Zhang, X.-K. Yang and K.-J. Huang, Colloids Surf., B, 2011, 83, 78-82.

76 D. Han, T. Han, C. Shan, A. Ivaska and L. Niu, Electroanalysis, 2010, 22, 2001-2008.

77 M. Li, J. E. Zhu, L. Zhang, X. Chen, H. Zhang, F. Zhang, S. Xu and D. G. Evans, Nanoscale, 2011, 3, 4240-4246.

78 Y. Zhang, R. Yuan, Y. Chai, W. Li, X. Zhong and H. Zhong, Biosens. Bioelectron., 2011, 26, 3977-3980.

79 M. Mallesha, R. Manjunatha, C. Nethravathi, G. S. Suresh, M. Rajamathi, J. S. Melo and T. V. Venkatesha, Bioelectrochemistry, 2011, 81, 104-108.

80 Z.-H. Sheng, X.-Q. Zheng, J.-Y. Xu, W.-J. Bao, F.-B. Wang and X.-H. Xia, Biosens. Bioelectron., 2012, 34, 125-131.

81 J.-H. Yang, D. Yang and Y. Li, Mater. Lett., 2014, 130, 111114.

82 A. Ambrosi and M. Pumera, Chem.-Eur. J., 2010, 16, 1094610949.

83 F. Gao, X. Cai, X. Wang, C. Gao, S. Liu, F. Gao and Q. Wang, Sens. Actuators, B, 2013, 186, 380-387.

84 Y.-R. Kim, S. Bong, Y.-J. Kang, Y. Yang, R. K. Mahajan, J. S. Kim and H. Kim, Biosens. Bioelectron., 2010, 25, 2366-2369.

85 H. Wang, F. Ren, R. Yue, C. Wang, C. Zhai and Y. Du, Colloids Surf., A, 2014, 448, 181-185.

86 B. Yang, H. Wang, J. Du, Y. Fu, P. Yang and Y. Du, Colloids Surf., A, 2014, 456, 146-152.

87 Z. Zhang, J. Yan, H. Jin and J. Yin, Electrochim. Acta, 2014, 139, 232-237.

88 M. A. Raj and S. A. John, J. Phys. Chem. C, 2013, 117, 43264335.

89 X. Yu, K. Sheng and G. Shi, Analyst, 2014, 139, 4525-4531.

90 S.-Q. Liu, W.-H. Sun and F.-T. Hu, Sens. Actuators, B, 2012, 173, 497-504.

91 T. Mary Nancy, V. Anithakumary and B. Kumara Swamy, J. Electroanal. Chem., 2014, 720, 107-114.

92 J. Ping, J. Wu, Y. Wang and Y. Ying, Biosens. Bioelectron., 2012, 34, 70-76.

93 S. Huang, H. Yue, J. Zhou, J. Zhang, C. Zhang, X. Gao and J. Chang, Electroanalysis, 2014, 26, 184-190.

94 B. Yu, D. Kuang, S. Liu, C. Liu and T. Zhang, Sens. Actuators, $B, 2014$, 205, 120-126.

95 N. G. Shang, P. Papakonstantinou, M. McMullan, M. Chu, A. Stamboulis, A. Potenza, S. S. Dhesi and H. Marchetto, Adv. Funct. Mater., 2008, 18, 3506-3514.

96 M. Zhang, C. Liao, Y. Yao, Z. Liu, F. Gong and F. Yan, Adv. Funct. Mater., 2014, 24, 978-985.
97 D. Usachov, O. Vilkov, A. Gruneis, D. Haberer, A. Fedorov, V. Adamchuk, A. Preobrajenski, P. Dudin, A. Barinov and M. Oehzelt, Nano Lett., 2011, 11, 5401-5407.

98 R. Lv and M. Terrones, Mater. Lett., 2012, 78, 209-218.

99 H. Liu, Y. Liu and D. Zhu, J. Mater. Chem., 2011, 21, 33353345.

100 Y. Wang, Y. Shao, D. W. Matson, J. Li and Y. Lin, ACS Nano, 2010, 4, 1790-1798.

101 S.-M. Li, S.-Y. Yang, Y.-S. Wang, C.-H. Lien, H.-W. Tien, S.-T. Hsiao, W.-H. Liao, H.-P. Tsai, C.-L. Chang and C.-C. M. Ma, Carbon, 2013, 59, 418-429.

102 N. Li, E. Zheng, X. Chen, S. Sun, C. You, Y. Ruan and X. Weng, Int. J. Electrochem. Sci., 2013, 8, 6524-6534.

103 C. Shan, H. Yang, D. Han, Q. Zhang, A. Ivaska and L. Niu, Langmuir, 2009, 25, 12030-12033.

104 Y. Si and E. T. Samulski, Nano Lett., 2008, 8, 1679-1682.

105 L. Wu, L. Feng, J. Ren and X. Qu, Biosens. Bioelectron., 2012, 34, 57-62.

106 S.-J. Li, J.-Z. He, M.-J. Zhang, R.-X. Zhang, X.-L. Lv, S.-H. Li and H. Pang, Electrochim. Acta, 2013, 102, 58-65.

107 H. S. Han, H. Seol, D. H. Kang, M. S. Ahmed, J.-M. You and S. Jeon, Sens. Actuators, B, 2014, 204, 289-296.

108 Q. Lian, Z. He, Q. He, A. Luo, K. Yan, D. Zhang, X. Lu and X. Zhou, Anal. Chim. Acta, 2014, 823, 32-39.

109 W. Zhang, Y. Chai, R. Yuan, S. Chen, J. Han and D. Yuan, Anal. Chim. Acta, 2012, 756, 7-12.

110 C. Wang, P. Xu and K. Zhuo, Electroanalysis, 2014, 26, 191198.

111 M. Liu, L. Wang, J. Deng, Q. Chen, Y. Li, Y. Zhang, H. Li and S. Yao, Analyst, 2012, 137, 4577-4583.

112 Y. Wang, W. Peng, L. Liu, M. Tang, F. Gao and M. Li, Microchim. Acta, 2011, 174, 41-46.

113 J. Li, J. Yang, Z. Yang, Y. Li, S. Yu, Q. Xu and X. Hu, Anal. Methods, 2012, 4, 1725-1728.

114 J. Yang, J. R. Strickler and S. Gunasekaran, Nanoscale, 2012, 4, 4594-4602.

115 S.-J. Li, D.-H. Deng, Q. Shi and S.-R. Liu, Microchim. Acta, 2012, 177, 325-331.

116 X. Tian, C. Cheng, H. Yuan, J. Du, D. Xiao, S. Xie and M. M. Choi, Talanta, 2012, 93, 79-85.

117 W. Zhu, T. Chen, X. Ma, H. Ma and S. Chen, Colloids Surf., $B, 2013,111,321-326$.

118 S. Liu, J. Yan, G. He, D. Zhong, J. Chen, L. Shi, X. Zhou and H. Jiang, J. Electroanal. Chem., 2012, 672, 40-44.

119 V. K. Ponnusamy, V. Mani, S.-M. Chen, W.-T. Huang and J. Jen, Talanta, 2014, 120, 148-157.

120 X. Liu, L. Xie and H. Li, J. Electroanal. Chem., 2012, 682, 158-163.

121 T. Qian, C. Yu, X. Zhou, S. Wu and J. Shen, Sens. Actuators, $B, 2014$, 193, 759-763.

122 T. Qian, C. Yu, S. Wu and J. Shen, Colloids Surf., B, 2013, 112, 310-314.

123 B. Kaur, T. Pandiyan, B. Satpati and R. Srivastava, Colloids Surf., B, 2013, 111, 97-106.

124 F. H. Cincotto, T. C. Canevari, A. M. Campos, R. Landers and S. A. Machado, Analyst, 2014, 139, 4634-4640. 
125 T.-Q. Xu, Q.-L. Zhang, J.-N. Zheng, Z.-Y. Lv, J. Wei, A.-J. Wang and J.-J. Feng, Electrochim. Acta, 2014, 115, 109-115.

126 W.-L. Liu, C. Li, L. Tang, Y. Gu and Z.-Q. Zhang, Chin. J. Anal. Chem., 2013, 41, 714-718.

127 C.-L. Sun, H.-H. Lee, J.-M. Yang and C.-C. Wu, Biosens. Bioelectron., 2011, 26, 3450-3455.

128 S. Palanisamy, S. Ku and S.-M. Chen, Microchim. Acta, 2013, 180, 1037-1042.

129 M. Rajkumar, B. Devadas, S.-M. Chen and P.-C. Yeh, Colloids Surf., A, 2014, 452, 39-45.

130 C.-H. Wu, C.-H. Wang, M.-T. Lee and J.-K. Chang, J. Mater. Chem., 2012, 22, 21466-21471.

131 J. Yan, S. Liu, Z. Zhang, G. He, P. Zhou, H. Liang, L. Tian, X. Zhou and H. Jiang, Colloids Surf., B, 2013, 111, 392-397. 132 J. Jiang and X. Du, Nanoscale, 2014, 6, 11303-11309.

133 C.-X. Xu, K.-J. Huang, Y. Fan, Z.-W. Wu, J. Li and T. Gan, Mater. Sci. Eng., C, 2012, 32, 969-974.

134 G. S. H. Thien, A. Pandikumar, N. M. Huang and H. N. Lim, Sci. Rep., 2014, 4, 5044.

$135 \mathrm{H}$. Teymourian, A. Salimi and S. Khezrian, Biosens. Bioelectron., 2013, 49, 1-8.

136 D. Wu, Y. Li, Y. Zhang, P. Wang, Q. Wei and B. Du, Electrochim. Acta, 2014, 116, 244-249.

137 J. Salamon, Y. Sathishkumar, K. Ramachandran, Y. S. Lee, D. J. Yoo, A. R. Kim and G. G. Kumar, Biosens. Bioelectron., 2015, 64, 269-276.

138 T. Peik-See, A. Pandikumar, H. Nay-Ming, L. Hong-Ngee and Y. Sulaiman, Sensors, 2014, 14, 15227-15243.

139 W. Sun, X. Wang, Y. Wang, X. Ju, L. Xu, G. Li and Z. Sun, Electrochim. Acta, 2013, 87, 317-322.

140 A. Yang, Y. Xue, Y. Zhang, X. Zhang, H. Zhao, X. Li, Y. He and Z. Yuan, J. Mater. Chem. B, 2013, 1, 1804-1811.

141 F. Zhang, Y. Li, Y.-e. Gu, Z. Wang and C. Wang, Microchim. Acta, 2011, 173, 103-109.

142 Y. Zeng, Y. Zhou, L. Kong, T. Zhou and G. Shi, Biosens. Bioelectron., 2013, 45, 25-33.

143 Y. Ma, M. Zhao, B. Cai, W. Wang, Z. Ye and J. Huang, Chem. Commun., 2014, 50, 11135-11138.

144 P. Nayak, P. Santhosh and S. Ramaprabhu, Graphene, 2013, 1, 25-30.

145 G. Inzelt, Conducting Polymers, Springer, 2008.

146 J. Janata and M. Josowicz, Nat. Mater., 2003, 2, 19-24.

147 Q. Liu, X. Zhu, Z. Huo, X. He, Y. Liang and M. Xu, Talanta, 2012, 97, 557-562.

148 W. Wang, G. Xu, X. T. Cui, G. Sheng and X. Luo, Biosens. Bioelectron., 2014, 58, 153-156.
149 W. Liu, J. Xiao, C. Wang, H. Yin, H. Xie and R. Cheng, Mater. Lett., 2013, 100, 70-73.

150 S. Liu, X. Xing, J. Yu, W. Lian, J. Li, M. Cui and J. Huang, Biosens. Bioelectron., 2012, 36, 186-191.

151 T. Qian, C. Yu, S. Wu and J. Shen, Biosens. Bioelectron., 2013, 50, 157-160.

152 H. S. Han, H. K. Lee, J.-M. You, H. Jeong and S. Jeon, Sens. Actuators, B, 2014, 190, 886-895.

153 X. Weng, Q. Cao, L. Liang, J. Chen, C. You, Y. Ruan, H. Lin and L. Wu, Talanta, 2013, 117, 359-365.

154 S. Hou, M. L. Kasner, S. Su, K. Patel and R. Cuellari, J. Phys. Chem. C, 2010, 114, 14915-14921.

155 L. Chang, Z. Jing, E. Yifeng, Y. Jingli, C. Lianshan and L. Donghui, Electron. J. Biotechnol., 2014, 17, 183-188.

156 S. Liu, B. Yu and T. Zhang, J. Mater. Chem. A, 2013, 1, 1331413320.

157 X. Liu, H. Zhu and X. Yang, RSC Adv., 2014, 4, 3706-3712.

158 X. Qin, H. Xiao-Ya and H. Shi-Rong, Electropolymerization, 2011, 4, 187-198.

159 C. Liao, M. Zhang, L. Niu, Z. Zheng and F. Yan, J. Mater. Chem. B, 2014, 2, 191-200.

160 S. Ijima, Nature, 1991, 354, 56-58.

161 X. Dong, Y. Ma, G. Zhu, Y. Huang, J. Wang, M. B. ChanPark, L. Wang, W. Huang and P. Chen, J. Mater. Chem., 2012, 22, 17044-17048.

162 C.-L. Sun, C.-T. Chang, H.-H. Lee, J. Zhou, J. Wang, T.-K. Sham and W.-F. Pong, ACS Nano, 2011, 5, 7788-7795.

163 S. Hu, Q. Huang, Y. Lin, C. Wei, H. Zhang, W. Zhang, Z. Guo, X. Bao, J. Shi and A. Hao, Electrochim. Acta, 2014, 130, 805-809.

164 X. Chen, J. Zhu, Q. Xi and W. Yang, Sens. Actuators, B, 2012, 161, 648-654.

165 S. Cheemalapati, S. Palanisamy, V. Mani and S.-M. Chen, Talanta, 2013, 117, 297-304.

166 Y. J. Yang and W. Li, Biosens. Bioelectron., 2014, 56, 300306.

167 S.-N. Ding, C.-L. Zheng, N. Wan and S. Cosnier, Monatsh. Chem. Chem. Mon., 2014, 1-6.

168 P. He, W. Wang, L. Du, F. Dong, Y. Deng and T. Zhang, Anal. Chim. Acta, 2012, 739, 25-30.

169 W. Wang, P. He, F. Dong, L. Du and T. Zhang, Anal. Chim. Acta, 2012, 739, 25-30.

170 X. Wang, Q. Wang, Q. Wang, F. Gao, F. Gao, Y. Yang and H. Guo, ACS Appl. Mater. Interfaces, 2014, 6, 1157311580. 